\title{
RETOUR VERS LE PASSÉ : LE PRINCIPE DE PRÉCAUTION ET LA RÉGULATION DES SUBSTANCES TOXIQUES AU CANADA ${ }^{()}$
}

\author{
HÉLÈNE TRUDEAU \\ Professeure agrégée \\ Centre de recherche en droit public. Université de Montréal \\ helene.trudeau@umontreal.ca \\ KATERI BEAULNE-BÉLISLE \\ Candidate à la maîtrise en sciences de l'environnement \\ Université du Québec à Montréal \\ kateribb@hotmail.com \\ THÉRÈSE LEROUX \\ Professeure titulaire \\ Centre de recherche en droit public. Université de Montréal \\ therese.leroux@umontreal.ca
}

Reçu: 27 avril 2010 / Accepté: 17 mai 2010

RÉSUMÉ : La dispersion croissante de substances toxiques dans l'environnement constitue une préoccupation importante pour le peuple canadien. La nécessité de protéger d'une part la population de l'Arctique contre les substances persistantes et

\footnotetext{
${ }^{(.)}$Recherche réalisée grâce à une subvention de la Commission du droit du Canada et de la Fondation pour la recherche juridique. En plus des organismes subventionnaires, nous remercions mesdames Carolina Monardes et Céline Nègre pour leur participation à cette étude.
} 
bioaccumulables, et d'autre part le bassin des Grands Lacs contre les rejets industriels abusifs, ont incité le gouvernement canadien à prendre des mesures pour davantage réglementer les produits chimiques dangereux. La Loi canadienne sur la protection de l'environnement de 1999 a ainsi créé l'obligation pour le gouvernement fédéral d'évaluer et de réduire les risques posés à l'environnement et/ou à la santé humaine par les 23000 substances qui ont été commercialisées au Canada. Dans cette procédure d'évaluation et de gestion à rebours des substances chimiques dangereuses, la Loi impose l'application du principe de précaution. L'inclusion de ce principe dans la Loi permettra-t-elle la prise de mesures efficaces lorsque les risques ne seront pas clairement étayés par les analyses scientifiques? La présente recherche suggère que pour jouer un rôle significatif dans les contextes d'incertitude scientifique, le principe de précaution tel que formulé dans la Loi devrait être renforcé par une réduction de la discrétion administrative et l'ajout d'obligations précises comme le renversement du fardeau de la preuve à l'encontre des promoteurs des substances, la prise en compte de facteurs de sécurité plus élevés dans l'évaluation des risques posés aux enfants et la fixation de délais pour l'établissement des stratégies de gestion de risques.

RESUM: La dispersió creixent de substàncies tòxiques en el medi ambient constitueix una preocupació important pel poble canadenc. La necessitat de protegir, d'una part, la població de l'Àrtic contra les substàncies persistents i bioacumulables i, de l'altra, la conca dels Grans Llacs contra els vessaments industrials abusius, han dut el govern canadenc a prendre mesures per regular els productes químics perillosos. La Llei canadenca de protecció del medi ambient de 1999 ha establert, d'aquesta manera, l'obligació per al govern federal d'avaluar i reduir els riscos per al medi ambient i/o la salut humana que representen les 23.000 substàncies que són comercialitzades al Canadà. Dintre d'aquest procediment d'avaluació i de gestió de les substàncies químiques perilloses, la Llei imposa l'aplicació del principi de precaució. La inclusió d'aquest principi dintre de la Llei permetrà la presa de mesures eficaces quan els riscos no hagin estat clarament definits per les anàlisis científiques? La recerca que aquí es presenta suggereix que, per jugar un paper significatiu dintre dels contextos d'incertesa científica, el principi de precaució, tal com està formulat a la Llei hauria d'ésser reforçat per una 
reducció de la discrecionalitat administrativa, tot afegint obligacions precises com la inversió de la càrrega de la prova en el cas dels promotors de les substàncies, la presa en compte de factors de seguretat més elevats en el cas de l'avaluació dels riscos imposats als infants, així com la fixació de moratòries per a l'establiment d'estratègies de gestió de riscos.

RESUMEN: La dispersión creciente de sustancias tóxicas en el medio ambiente constituye una preocupación importante para el pueblo canadiense. La necesidad de proteger, por una parte, la población del Ártico contra las sustancias persistentes y bioacumulabes, y, por la otra, la cuenca de los Grandes Lagos contra los vertidos industriales abusivos, han llevado al gobierno canadiense a tomar medidas para regular los productos químicos peligrosos. La Ley canadiense de protección del medio ambiente de 1999 ha establecido, de este modo, la obligación para el gobierno federal de evaluar y reducir los riesgos para el medio ambiente y/o la salud humana que suponen las 23.000 sustancias que son comercializadas en Canadá. Dentro de este procedimiento de evaluación y de gestión de las sustancias químicas peligrosas, la Ley impone la aplicación del principio de precaución ¿Permitirá la inclusión de este principio dentro de la Ley la toma de medidas eficaces cuando los riesgos no hayan sido claramente definidos por los análisis científicos? La investigación que aquí se presenta sugiere que, para jugar un papel significativo dentro de los contextos de incertidumbre científica, el principio de precaución, tal como está formulado en la Ley debería ser reforzado a través de una reducción de la discrecionalidad administrativa, añadiendo obligaciones precisas como la inversión de la carga de la prueba en el caso de los promotores de las sustancias, la toma en consideración de factores de seguridad más elevados en el caso de la evaluación de riesgos impuestos a los niños, así como la fijación de moratorias para el establecimiento de estrategias de gestión de riesgos.

ABSTRACT: The dispersion of toxic substances in the environment is a matter of serious concerns for Canadians. With the necessity to protect a Nordic population exposed to persistent and bioaccumulative pollutants migrating from southern regions and to assure 
the quality of the Great Lakes freshwater basin, the Canadian government appears determined to limit the manufacturing, selling and use of dangerous chemicals throughout the country. The Canadian Environmental Protection Act of 1999 has imposed on the federal government the duty to identify and reduce the risks posed to both the environment and the human health by some 23000 chemicals manufactured or imported within Canada in the past fifty years, often without any assessment as to their toxicity. The Act calls for the application of the precautionary principle by the government in this procedure. But will this principle as formulated in the Act prove to be sufficiently strong to protect the environment and the population when the risks involved will not be clearly determined by scientists? This present research suggests that to play a significant role in the management of the risks posed by toxic chemicals, the precautionary principle has to be rendered more «operational» in the context of the Canadian Environmental Protection Act. In particular, the Act should be modified to reduce the administrative discretion and to set mandatory actions when dealing with scientific uncertainties. This includes reversing the burden of proof over manufacturers for the assessment of the toxicity of certain chemicals, applying greater safety factors in the evaluation of risks to children and imposing delays in the establishment of risk management strategies.

MOTS CLÉS : Principe de précaution — substances toxiques — évaluation des risques

PARAULES CLAU: Principi de precaució — substàncies tòxiques — avaluació de riscos

PALABRAS CLAVE: Principio de precaución - sustancias tóxicas - evaluación de riesgos

KEYWORDS: Precautionary principle — toxic substances — risk assessment 
Sommaire : I. L'évaluation et la gestion des substances existantes : la Partie V de la L.C.P.E. 1. Le processus d'évaluation du risque écologique posé par les substances existantes. 1.1. Cadre et terminologie. 1.2. Principales étapes du processus d'évaluation des substances existantes. 2. La gestion des substances toxiques selon les paramètres prévus à la L.C.P.E. II. L'incertitude scientifique et sa prise en compte juridique dans le contexte de la L.C.P.E. 1. Le principe de précaution. 2. La méthode du poids de la preuve. III. Les paramètres d'application du principe de précaution dans l'évaluation et la gestion des substances toxiques : quelques pistes de réflexion. 1. Opérationnaliser le principe de précaution et diminuer la discrétion administrative. 2. Instaurer le principe de la responsabilité de l'industrie dans la démonstration de l'innocuité des substances. 3. Assurer la vérification de la preuve scientifique et l'indépendance des évaluateurs. IV. Conclusion. V. Bibliographie. 1. Doctrine juridique. 2. Documents et rapports gouvernementaux et d'organisations internationales. 3. Documents et rapports d'organisations non gouvernementales.

Sur la scène internationale, le Canada s'est souvent présenté comme étant à l'avant-garde dans la lutte contre les substances toxiques et l'élimination des plus dangereuses d'entre elles $^{1}$. De par sa situation géographique et l'immensité de son territoire, le Canada constitue l'un des pays du monde qui risquent le plus de souffrir des effets néfastes de l'accumulation des substances toxiques dans l'environnement. Certains écosystèmes et certains groupes de populations sont en effet affectés plus sévèrement que d'autres par la dispersion des produits chimiques. Les écosystèmes des Grands Lacs figurent parmi ceuxci, les nombreuses entreprises et municipalités qui en sont riveraines contribuant à en polluer les eaux de façon importante. Le bassin des Grands Lacs possède la plus grande superficie d'eau douce en surface au monde, quelque $245000 \mathrm{~km}^{2}$, ce qui représente près de $20 \%$ de la réserve planétaire en eau douce et $80 \%$ de celle de l'Amérique du Nord ${ }^{2}$. La Stratégie binationale relative aux toxiques des Grands Lacs, signée en 1997 par le Canada et les États-Unis, vise à concerter l'action de ces deux pays face à la menace que représentent de nombreuses substances toxiques et persistantes au sein de cet écosystème

\footnotetext{
${ }^{1}$ Par exemple, le gouvernement canadien affirme relativement au Plan de gestion des produits chimiques: "Le nouveau plan mettra à profit la position de chef de file mondial du Canada dans la saine gestion des substances et des produits chimiques". Gouvernement du Canada, «Plan de gestion des produits chimiques», en

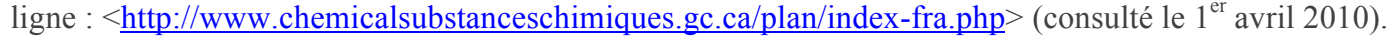

${ }^{2}$ Parcs Canada, « Aires marines nationales de conservation du Canada; Le Plan de réseau des aires marines nationales de conservation du Canada; Le milieu aquatique des Grands Lacs canadiens », en ligne :

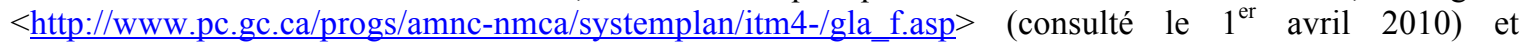
Environnement Canada, «La Stratégie binationale relative aux toxiques des Grands Lacs », en ligne : http://ec.gc.ca/cleanair-airpur/Branche_sur_l'air_pur _-

_Regions/Region_de l'Ontario/Interventions_gouvernementales/Strategie binationale_relative aux toxique s_des_Grands_Lacs-WS2FC703E8-1_Fr.htm> (consulté le $1^{\text {er }}$ avril 2010).
} 
essentiel $^{3}$. Sous 1'égide de la Commission mixte internationale, cette Stratégie établit notamment des objectifs communs ou individuels consentis par les deux États, l'objectif général consistant en la quasi-élimination des principales substances toxiques persistantes d'origine anthropique présentes dans le bassin des Grands Lacs ${ }^{4}$.

Au Canada, outre les écosystèmes et les populations des Grands Lacs, ceux des régions arctiques sont aussi particulièrement exposés aux substances toxiques, principalement aux polluants organiques persistants, communément appelés POP $^{5}$. Le préambule de la Convention de Stockholm sur les polluants organiques persistants ${ }^{6}$ comporte d'ailleurs le paragraphe suivant :

«Sachant que l'écosystème arctique et les populations autochtones qui y vivent sont particulièrement menacés en raison de la bio-amplification des polluants organiques persistants, et que la contamination des aliments traditionnels de ces populations constitue une question de santé publique.»

Des concentrations élevées de POP sont en effet observées chez les prédateurs du plus haut niveau trophique vivant dans le nord du Canada. Ces substances sont généralement

3 Environnement Canada et U. S. Environmental Protection Agency (EPA), « La Stratégie binationale

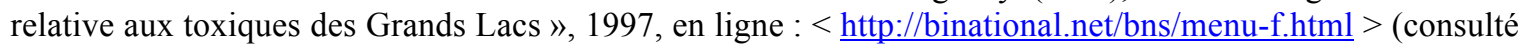
le 1 er avril 2010). Cette Stratégie découle de l'Accord relatif à la qualité de l'eau dans les Grands Lacs et plus particulièrement du Septième rapport biennal sur la qualité de l'eau dans les Grands Lacs (1994) de la Commission mixte internationale. Voir Commission mixte internationale, «L'Accord relatif à la qualité de l'eau dans les Grands Lacs », 1978, en ligne : http://www.ijc.org/rel/agree/fquality.html\#agrm (consulté le $1^{\text {er }}$ avril 2010) et Commission mixte internationale, " Septième rapport biennal en vertu de l'Accord de 1978 relatif à la qualité de l'eau dans les Grands Lacs », 1994, en ligne : $<$ http://www.ijc.org/php/publications/html/7brf.html $>$ (consulté le $1^{\mathrm{er}}$ avril 2010).

${ }^{4}$ Environnement Canada et U. S. Environmental Protection Agency (EPA), « La Stratégie binationale

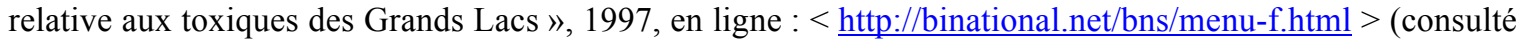
le 1er avril 2010).

5 Les polluants organiques persistants comprennent des produits chimiques industriels comme les BPC, des pesticides comme le DDT, le chlordane et le toxaphène, ainsi que des contaminants comme les dioxines et les furannes. Environnement Canada, en ligne : < http://www.ec.gc.ca/cleanairairpur/default.asp?lang=Fr\&n=8F6FD286-1 > (consulté le 1er avril 2010).

${ }^{6}$ Le Canada est devenu en 2001 le premier pays à ratifier la Convention de Stockholm, qui est entrée en vigueur le 17 mai 2004. Secretariat of the Stockholm Convention, «Convention de Stockholm sur les polluants organiques persistants », 22 mai 2001, en ligne:

$<$ http://chm.pops.int/Portals/0/Repository/convention text/UNEP-POPS-COP-CONVTEXT-

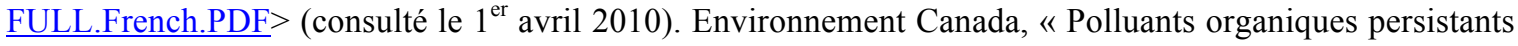
(POP) », en ligne: 
transportées au nord par des processus atmosphériques et s'accumulent dans les tissus des organismes en des concentrations croissantes tout au long de la chaîne alimentaire. La faune comme les populations nordiques peuvent donc être exposées aux effets biologiques de ces substances.

On comprend dès lors qu'une part importante des démarches politiques canadiennes en matière d'évaluation et de gestion des substances toxiques découle des préoccupations soulevées au pays face à la contamination croissante des Grands Lacs et de l'environnement arctique ${ }^{7}$. Plus récemment, les révélations médiatiques à l'effet que des échantillons de sang provenant de la population canadienne en général contiennent un impressionnant cocktail de substances chimiques ont alerté l'opinion publique ${ }^{8}$.

Alors que se poursuit au niveau mondial la mobilisation des États en vue d'éliminer progressivement les principaux polluants organiques persistants ${ }^{9}$, le Canada a entrepris de son côté un exercice de très longue haleine visant l'évaluation et la gestion des risques

$<$ http://www.ec.gc.ca/cleanair-airpur/Polluants/Polluants_organiques_persistants_(POP)-WS8F6FD286-

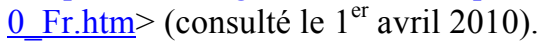

${ }^{7}$ Des incidents survenus dans les années 80 ont aussi contribué à accroître les préoccupations publiques par rapport aux substances toxiques, comme la contamination chimique provoquée par des fuites au site d'enfouissement de Love Canal, près de Niagara Falls, dans l'État de New York et la découverte de résidus toxiques au fond de la rivière Sainte-Claire, située près de Windsor, en Ontario. Environnement Canada, «Au sujet de l'ancienne LCPE », en ligne:

$<$ http://www.ec.gc.ca/RegistreLCPE/gene_info/CEPA1988.cfm> (consulté le $1^{\text {er }}$ avril 2010).

${ }^{8}$ Les études réalisées en 2005 et 2006 par l'association Défense environnementale ont porté sur les populations canadiennes adulte et enfant : Défense environnementale, «Une nation toxique : Rapport sur la pollution chez les Canadiens », 2005,

$<$ http://www.toxicnation.ca/files/toxicnation/report/PATN_FrenchWeb.pdf $>$ (consulté le $1^{\text {er }}$ avril 2010) et Défense environnementale, «Des enfants empoisonnés - une nation toxique : Rapport sur la pollution chez les familles canadiennes», 2006, <http://www.toxicnation.ca/files/toxicnation/report/PCTN French.pdf $>$ (consulté le $1^{\text {er }}$ avril 2010). Quatre politiciens fédéraux se sont même livrés au jeu et leurs échantillons de sang analysés ont aussi révélé une contamination par de nombreux polluants dangereux: Défense environnementale, «Une nation toxique sur la Colline du Parlement: Rapport sur la pollution chez quatre politiciens canadiens», 2007, en ligne : <http://www.environmentaldefence.ca/toxicnation/report/TNPARLIAMENTHILL fr.pdf> (consulté le $1^{\text {er }}$ avril 2010).

${ }^{9}$ En mai 2009, la Conférence des Parties à la Convention de Stockholm sur les polluants organiques persistants a ajouté 9 substances aux listes visant la réduction et l'élimination progressive des POP : Stockholm Convention Secretariat, «Les gouvernements s'unissent afin d'intensifier leurs efforts pour réduire la dépendance mondiale au DDT et ajouter neuf produits chimiques à la liste existante d'un traité international », Communiqué de presse, 9 mai 2009, en ligne :

$<$ http://chm.pops.int/Convention/Media/Pressreleases/COP4Geneva9May2009/tabid/542/language/fr-

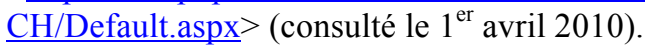


posés par les milliers de substances fabriquées, importées ou commercialisées en sol canadien. Fort de la compétence constitutionnelle que la Cour suprême du Canada lui a reconnue en la matière en $1997^{10}$, le gouvernement canadien entend prendre des mesures pour contrer les risques liés aux plus dangereuses d'entre elles, notamment en procédant à leur quasi-élimination. Ainsi, en septembre 2006, le gouvernement fédéral menait à terme un exercice de catégorisation des 23000 substances figurant à la Liste intérieure des substances ${ }^{11}$ établie en vertu de la Loi canadienne sur la protection de l'environnement ${ }^{12}$. Selon les résultats obtenus par ce processus, plus de 4000 substances devront faire l'objet d'une évaluation plus poussée des risques qu'elles peuvent présenter pour l'environnement et/ou la santé humaine. L'évaluation de près de 200 d'entre elles a été identifiée comme étant hautement prioritaire. Le Plan de gestion des substances chimiques du Canada, dévoilé en décembre 2006, établit la stratégie préconisée par le gouvernement canadien à l'endroit des substances qui auront été considérées toxiques ${ }^{13}$.

C'est à cet exercice de «retour vers le passé» que la présente étude s'intéresse. Il est encore trop tôt pour présenter un bilan de celui-ci, l'évaluation des substances catégorisées étant à peine entamée et l'établissement de stratégies de gestion des risques pour les substances visées pouvant fort bien s'étendre au-delà de l'année 2020. Une question essentielle se pose cependant : ce travail entrepris par le gouvernement fédéral à l'endroit des substances commercialisées au Canada permettra-t-il d'identifier et de

\footnotetext{
${ }^{10}$ R. c. Hydro-Québec, [1997] 3 R.C.S. 213. Dans un jugement très divisé, la Cour suprême du Canada a décidé que le régime législatif adopté par le gouvernement fédéral concernant la réglementation des substances toxiques était justifié en vertu de la compétence fédérale en matière de droit criminel, et qu'il était donc constitutionnel. Il est important de noter que par l'application de la théorie constitutionnelle du double aspect, les provinces peuvent elles aussi réglementer les substances toxiques en vertu des larges compétences qu'elles détiennent sur leurs territoires, en autant que ces mesures provinciales ne soient pas en conflit avec les mesures fédérales.

${ }^{11}$ Ci-après L.I.S.

${ }^{12}$ Art. 73 Loi canadienne sur la protection de l'environnement, L.R.C. 1999, c. 33 (Ci-après L.C.P.E.), En vertu de l'art. 66 de la Loi, le ministre de l'Environnement a l'obligation de tenir à jour la Liste intérieure des substances, soit la liste des substances qui ont été soit fabriquées ou importées au Canada par une personne en une quantité d'au moins $100 \mathrm{~kg}$ au cours d'une année civile entre le $1^{\text {er }}$ janvier 1984 et le 31 décembre 1986, soit commercialisées ou utilisées à des fins de fabrication commerciale au Canada pendant cette période. Cette liste contient aussi les substances fabriquées, importées ou commercialisées au Canada depuis 1987, ajoutées notamment au moyen du régime applicable aux substances nouvelles depuis 1994 : voir art. 80 et ss. L.C.P.E.
}

13 Gouvernement du Canada, «Plan de gestion des produits chimiques», en ligne : $<$ http://www.chemicalsubstanceschimiques.gc.ca/plan/index-fra.php> (consulté le 1er avril 2010). 
contrôler adéquatement les substances chimiques pour lesquelles le risque scientifique demeure incertain? L'enjeu est ici de taille et l'exercice entrepris pourrait s'avérer vain et coûteux s'il ne mène pas à une meilleure prise en compte par les pouvoirs politiques de l'incertitude scientifique à l'égard des risques. En effet, l'absence même d'un quelconque processus visant à identifier les risques potentiels, a conduit par le passé à la commercialisation de substances qui se sont avérées par la suite dangereuses. Le législateur a-t-il su introduire, dans la L.C.P.E. de 1999, suffisamment de dispositifs pour assurer que les pouvoirs publics puissent dorénavant protéger la population canadienne dans les situations d'incertitude scientifique? De façon plus précise, le principe de précaution, qui est devenu l'outil juridique de choix pour se prévaloir à l'endroit des risques incertains, sera-t-il appliqué par le gouvernement canadien dans l'évaluation et la gestion des risques posés par les substances chimiques commercialisées au Canada?

Pour répondre à cette question, le présent texte sera divisé en trois parties. Nous verrons successivement le processus d'évaluation et de gestion des substances existantes instauré par la Partie V de la L.C.P.E. (1), l'incertitude scientifique et sa prise en compte dans le contexte de cette Loi (2), pour finalement suggérer quelques pistes de réflexion pour favoriser l'application du principe de précaution dans l'évaluation et la gestion des substances toxiques (3).

\section{L'ÉVALUATION ET LA GESTION DES SUBSTANCES EXISTANTES : LA PARTIE V DE LA 'L.C.P.E.'}

L'intervention du gouvernement fédéral en matière de contrôle des produits chimiques s'est accrue au Canada à partir des années 1980. Des pas importants ont été franchis, d'abord avec l'adoption de la Loi canadienne sur la protection de l'environnement de $1988^{14}$ qui prévoyait les premières mesures visant la réglementation des substances toxiques «du berceau au tombeau» ${ }^{15}$. La L.C.P.E. de 1988 a ainsi permis l'inscription d'une quarantaine de substances à l'Annexe I, comprenant la liste des substances

\footnotetext{
14 L.R.C. (1985), ch. 16 (4e suppl.).

15 Art. 34 L.C.P.E (1988).
} 
considérées toxiques au terme d'une évaluation des risques. Cette liste reconduite par la L.C.P.E. de 1999 en contient maintenant près d'une centaine. Les ajouts de substances se feront désormais à un rythme accéléré puisque seront réalisées des évaluations préalables suivant le processus de catégorisation des 23000 substances figurant à la L.I.S., qui devraient conduire à la réglementation et à la gestion de centaines d'entre elles dans les prochaines décennies. Par ailleurs, l'année 1994 a marqué aussi une étape importante, puisque depuis cette date toutes les nouvelles substances introduites au Canada doivent faire l'objet de tests prescrits en vertu du Règlement sur les renseignements concernant les substances nouvelles (substances chimiques et polymères) ${ }^{16}$. Auparavant, aucune évaluation des risques n'était réalisée au moment de l'introduction des substances, de leur fabrication ou de leur commercialisation en sol canadien.

Plusieurs programmes et organismes du gouvernement du Canada visent à réguler les substances chimiques ${ }^{17}$. Or, la présente étude s'intéresse au contrôle des substances soumises à la Partie $\mathrm{V}$ de la L.C.P.E., qui est exercé conjointement par le ministre fédéral de l'Environnement et le ministre fédéral de la Santé ${ }^{18}$. Notre analyse se limitera cependant à la prise en compte de l'incertitude scientifique et à l'application du principe

${ }^{16}$ DORS/2005-247 et DORS/2005-248. DORS/94-260, abrogé. Le règlement de 1994 comportait des dispositions transitoires visant les substances introduites au Canada entre janvier 1987 et juillet 1994.

${ }^{17}$ En effet, la partie $\mathrm{V}$ de la L.C.P.E., qui fait l'objet du présent texte, joue à l'égard des substances chimiques un rôle résiduaire, en ce sens que s'il existe un autre régime législatif fédéral qui prévoit à l'endroit de catégories données de substances un préavis de fabrication, d'importation ou de vente et une évaluation en vue de déterminer si ces substances sont effectivement ou potentiellement toxiques, ce régime s'appliquera plutôt que celui prévu par la L.C.P.E. : voir l'alinéa 81 (6) a) L.C.P.E. C'est le gouverneur en conseil qui décide si les conditions d'application de cet alinéa sont réunies relativement à une loi fédérale ou à ses règlements d'application. Il peut par décret inscrire une loi et ses règlements sur la liste de l'annexe 2 de la Loi, si les conditions sont réunies, et les radier de la liste, si les conditions ne sont plus réunies (alinéas 81 (7) a) et b)). À l'heure actuelle, figurent sur la liste de l'annexe 2 de la L.C.P.E., la Loi sur les produits antiparasitaires, la Loi sur les engrais et la Loi relative aux aliments du bétail, de même que leurs règlements d'application. Les nouvelles substances qui répondent aux définitions proposées par ces lois ne sont pas assujetties à la procédure d'évaluation prévue à la L.C.P.E., mais à un régime établi par ces dernières lois.

${ }^{18}$ Les processus d'évaluation des substances par Environnement Canada et Santé Canada, bien qu'étant effectués de façon concertée, ne se recoupent pas. Environnement Canada applique des paramètres d'évaluation permettant d'apprécier les risques que pose pour les organismes vivants autres qu'humains l'exposition à une substance. Le ministère de la Santé évalue de son côté les effets de l'exposition aux substances chimiques pour l'être humain, en vertu de paramètres différents, qui cherchent par exemple à apprécier le caractère cancérigène d'une substance. La conclusion d'un seul des deux ministères à l'effet qu'une substance est effectivement ou potentiellement toxique est suffisante pour entraîner l'inscription de celle-ci à la liste des substances toxiques, sans que cette conclusion ne puisse être remise en cause par le ministère effectuant l'autre volet de l'évaluation. 
de précaution dans le cadre du Programme des substances chimiques d'Environnement Canada ${ }^{19}$.

Le contrôle d'une substance exercé en vertu de la partie $\mathrm{V}$ de la L.C.P.E., comprend deux phases principales, celle de l'évaluation de sa toxicité potentielle, puis celle de sa gestion, le cas échéant. Une équipe d'Environnement Canada est chargée de l'évaluation des substances dites existantes, une autre de celle des substances dites nouvelles. Une dernière équipe est quant à elle responsable de leur gestion ${ }^{20}$. Notre étude s'intéressant principalement aux outils juridiques mis en place par les équipes d'Environnement Canada pour corriger les erreurs du passé, à savoir pour correctement évaluer et gérer les risques éventuels posés par des substances déjà commercialisées, nos propos concerneront davantage les substances existantes que les substances nouvelles. Nous verrons donc successivement dans cette première partie le processus d'évaluation du risque écologique posé par les substances existantes (1.1) et la gestion des substances toxiques selon les paramètres prévus à la L.C.P.E. (1.2).

\section{Le processus d'évaluation du risque écologique posé par les substances existantes}

\subsection{Cadre et terminologie}

Le terme «substance» reçoit dans la L.C.P.E. une acception très large. Il vise, sauf dans quelques cas, «toute matière organique ou inorganique, animée ou inanimée, distinguable» ${ }^{21}$.

L'évaluation d'une substance vise à déterminer si celle-ci est toxique ou potentiellement toxique au sens de la L.C.P.E. En vertu de l'article 64 de cette loi :

\footnotetext{
${ }^{19}$ Le projet de recherche qui est à l'origine de la présente étude porte effectivement sur l'application du principe de précaution dans l'évaluation et la gestion du risque écologique au sein du gouvernement canadien. Le présent texte s'appuie d'ailleurs en partie sur les résultats d'entretiens semi-dirigés menés auprès de fonctionnaires du Programme des substances existantes d'Environnement Canada que nous tenons à remercier pour leur disponibilité et l'accueil chaleureux qu'ils nous ont réservés.

${ }^{20}$ Les articles applicables plus particulièrement aux substances existantes et aux substances nouvelles sont respectivement les suivants : 73 à 79 et 80 à 89 L.C.P.E. Les articles 90 à 94 L.C.P.E. quant à eux visent la réglementation des substances toxiques. D'autres articles de la L.C.P.E. concernent également des instruments de gestion des substances, notamment les articles 10, 54-60, 93 et 196-200.

${ }^{21}$ Art. 3 L.C.P.E.
} 
« [...] est toxique toute substance qui pénètre ou peut pénétrer dans l'environnement en une quantité ou concentration ou dans des conditions de nature à :

a) avoir, immédiatement ou à long terme, un effet nocif sur l'environnement ou sur la diversité biologique;

b) mettre en danger l'environnement essentiel pour la vie;

c) constituer un danger au Canada pour la vie ou la santé humaines.»

Cette définition du terme «toxique» inclut à la fois l'exposition à une substance et l'évaluation des dangers qu'elle présente. Elle correspond à une approche d'évaluation des risques (exposition et danger), qu'il faut distinguer d'une approche d'évaluation du danger (ou de la toxicité intrinsèque). La première approche reflète l'idée que c'est la dose qui fait le poison : tout peut être néfaste pour les organismes vivants en une quantité suffisante. L'approche d'évaluation du danger se réfère plutôt à une idée de toxicité « intrinsèque », c'est-à-dire qu'une substance peut se révéler plus dangereuse que d'autres à concentrations égales pour des organismes vivants. Ce sont souvent des tests en laboratoire qui permettent de constater ces effets négatifs à divers niveaux de concentration d'une substance. La prise en considération de données factuelles sur l'exposition confère plus de précision et de justesse à l'évaluation scientifique de la toxicité des substances. Elle rend toutefois nettement plus long et ardu le processus d'évaluation. En effet, généralement, les données d'exposition aux substances sont inexistantes ou très incomplètes et sont difficiles à recueillir ${ }^{22}$. Notons également que le fait de procéder à l'évaluation de la toxicité intrinsèque de chaque substance individuellement, plutôt que par familles de substances de composition chimique similaire, a aussi pour effet d'accroître le degré de certitude qui lui est associée, sans compter que cela semble convenir à l'interprétation judiciaire des pouvoirs fédéraux en matière de contrôle des substances toxiques ${ }^{23}$. Cela prolonge et complexifie cependant les démarches visant à identifier les substances toxiques au sens de la Loi.

\footnotetext{
${ }^{22}$ Ces données concernent trois volets distincts : les entrées de la substance dans l'environnement, son devenir et les expositions à celle-ci.

${ }^{23}$ Voir R. c. Hydro-Québec, [1997] 3 R.C.S. 213.
} 
Les expressions «substances existantes» et «substances nouvelles» ne sont pas définies dans la L.C.P.E. La compréhension de ce que recouvrent ces termes et de ce qui les distingue apparaît pourtant essentielle pour saisir la globalité du rôle joué par le gouvernement canadien dans l'évaluation et la gestion des substances toxiques.

Au sujet des substances nouvelles, le gouvernement mentionne:

«Une substance nouvelle est une substance pour laquelle une déclaration est nécessaire avant qu'elle ne soit importée ou fabriquée. Les substances nouvelles sont :

- Celles qui ne sont pas inscrites sur la Liste intérieure des substances (LIS). Il y a certaines exemptions $[\ldots]$

- Les substances $[\ldots]$ proposées pour une nouvelle activité

- Les polymères $[\ldots]$ pour lesquels la version non ERR (Polymères à exigences réglementaires réduites) du polymère est proposée pour l'importation ou la fabrication» ${ }^{24}$

Le Programme des substances nouvelles permet de cibler les substances qui pourraient s'avérer toxiques avant même qu'elles ne soient fabriquées ou introduites en sol canadien et d'en éviter ainsi la dispersion ou encore d'en limiter l'utilisation. L'évaluation d'une substance qui ne figure pas à la Liste intérieure de substances et que l'on cherche à introduire ou à fabriquer au Canada au-delà d'une certaine quantité déterminée est donc obligatoire. $\mathrm{Si}$, au terme de cette évaluation, l'importation ou la fabrication de la nouvelle substance est autorisée, celle-ci est ajoutée à la L.I.S.

Les substances existantes sont celles qui sont ou qui ont été fabriquées, importées, commercialisées ou utilisées à des fins commerciales en sol canadien, et celles qui sont ou ont été rejetées dans l'environnement canadien ${ }^{25}$. Elles sont donc de deux types: les rejets

\footnotetext{
${ }^{24}$ Gouvernement du Canada, «Qui doit déclarer et comment », en ligne :

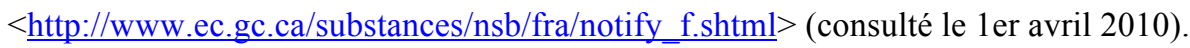

${ }^{25}$ Environnement Canada, « La Loi canadienne sur la protection de l'environnement, 1999 (LCPE 1999) et l'évaluation des substances actuelles », en ligne :

$<\underline{\text { http://www.ec.gc.ca/RegistreLCPE/gene info/fact } 08 . c f m}>$ (consulté le $1^{\text {er }}$ avril 2010).
} 
de polluants, essentiellement le produit de l'activité industrielle qui a cours au pays, et les substances commerciales qui apparaissent à la L.I.S. Même s'il s'avère impossible de répertorier la totalité des rejets industriels au Canada, un grand nombre de ceux-ci figurent à 1'Inventaire national des rejets de polluants (I.N.R.P.) ${ }^{26}$ qui est mis à jour chaque année ${ }^{27}$.

Le processus d'évaluation des substances existantes, qu'il s'agisse de rejets industriels ou de substances commercialisées, s'avère nécessairement long et coûteux, compte tenu de la complexité de la tâche et du nombre pratiquement incalculable de composés et mélanges chimiques qui sont utilisés ou déversés sous une forme ou une autre dans l'environnement. Il faut comprendre qu'à l'endroit des substances existantes, la L.C.P.E. de 1999 prévoit deux mécanismes distincts menant à l'évaluation. Depuis l'adoption de la L.C.P.E. de 1988, il y a un processus «régulier» d'évaluation qui peut viser autant les rejets industriels que les substances commerciales et qui est exercé en fonction des priorités d'intervention du gouvernement et des ressources budgétaires disponibles. Ce processus régulier n'a assuré au fil des années l'évaluation et la gestion que d'un nombre très restreint de substances ${ }^{28}$. La L.C.P.E. de 1999 a aussi instauré un mécanisme de révision (la «catégorisation») des 23000 substances commercialisées au Canada (et non des rejets industriels) qui n'ont pas antérieurement fait l'objet d'une évaluation des risques qu'elles présentaient. La Loi prévoit que cette catégorisation sera suivie d'une

\footnotetext{
${ }^{26}$ Établi en vertu de l'article 48 L.C.P.E.

${ }^{27}$ Voir le site internet d'Environnement Canada, «Inventaire national des rejets de polluants», en ligne : $<$ http://www.ec.gc.ca/inrp-npri/default.asp?lang=Fr\&n=4A577BB9-1 $>$ (consulté le $1^{\text {er }}$ avril 2010).

${ }^{28}$ «Certaines substances avaient été élaborées et mises en marché avant que la recherche toxicologique détaillée ne soit pratique courante, alors que d'autres avaient été largement utilisées à des fins commerciales pendant des décennies. Par conséquent, l'approche adoptée au Canada et par d'autres compétences était axée sur les risques liés aux polluants utilisés à grande échelle, notamment les effluents de combustion, comme les dioxines et les furannes, ou les rejets dans l'eau, comme les effluents des usines ou d'autres installations. Le gouvernement chargeait des groupes de scientifiques d'identifier les polluants susceptibles d'être dangereux, pour ensuite évaluer les risques qu'ils posaient pour l'environnement et la santé. Dans la plupart des cas, si le processus d'évaluation permettait de déterminer que la substance posait un risque inacceptable pour l'environnement ou la santé humaine, le gouvernement inscrivait la substance sur la Liste des substances toxiques de la LCPE et mettait en œuvre des mesures pour prévenir ou gérer les risques posés par ces substances» : Gouvernement du Canada, L'examen de la Loi canadienne sur la protection de l'environnement (1999); Réponse provisoire $d u$ gouvernement, octobre 2007, en ligne :

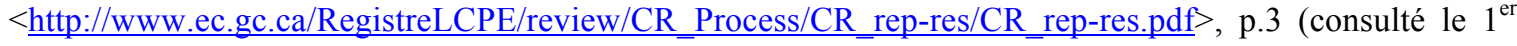
avril 2010).
} 
évaluation complète (appelée dans le cadre de la Loi «évaluation préalable ${ }^{29}$ ») pour les substances qui possèdent certaines caractéristiques : celles qui soit présentent pour les particuliers au Canada le plus fort risque d'exposition, soit sont persistantes ou bioaccumulables au sens des règlements ${ }^{30}$ et présentent, d'après des études en laboratoire ou autres, une toxicité intrinsèque ${ }^{31}$ pour les êtres humains ou les organismes autres que les organismes humains ${ }^{32}$.

\subsection{Principales étapes du processus d'évaluation des substances existantes}

En vertu de la L.C.P.E. de 1988, les substances pour lesquelles les ministres jugeaient prioritaire de déterminer si elles étaient effectivement ou potentiellement toxiques se voyaient inscrites à la liste des substances d'intérêt prioritaire en vigueur (L.S.I.P. 1 ou L.S.I.P. $2^{33}$ ) et faisaient l'objet d'une évaluation s'étendant souvent sur plusieurs années. Ce processus constituait à l'époque le seul moyen de soumettre une substance à une évaluation de risques et a été grandement critiqué, en raison de sa lenteur et de son

\footnotetext{
${ }^{29}$ Art. 74 L.C.P.E.

${ }^{30}$ Règlement sur la persistance et la bioaccumulation, DORS/2000-107.

31 «Capacité intrinsèque d'une substance de causer du tort. Cette toxicité est le plus souvent déterminée à partir des résultats d'analyses en laboratoire. Par conséquent, une substance peut être considérée comme intrinsèquement toxique, peu importe qu'il y ait exposition ou non. La toxicité intrinsèque joue un rôle très important lorsqu'il s'agit d'établir les niveaux d'exposition auxquels surviennent des effets nocifs; conjuguée aux renseignements sur l'exposition, elle est un facteur déterminant dans l'évaluation des risques ॥; Gouvernement du Canada, Mesures législatives sur la protection de l'environnement conçues pour l'avenir - Une LCPE renouvelée, Ottawa: Ministre des Approvisionnements et Services, 1995, pp. 7879.

${ }^{32}$ Art. 73 L.C.P.E.

${ }^{33}$ La mise en œuvre du Programme d'évaluation des substances d'intérêt prioritaire a débuté avec l'adoption de la L.C.P.E. (1988). En vertu de cette loi, les ministres de la Santé et de l'Environnement devaient élaborer une liste de substances d'intérêt prioritaire pour ensuite évaluer leur toxicité. Ainsi, une première liste comprenant 44 substances d'intérêt prioritaire (L.S.I.P.1) a été publiée en 1989 à la Gazette du Canada. L'évaluation de ces substances a pris fin en février 1994. Parmi ces substances, 24 ont été considérées toxiques et l'évaluation de 7 autres est demeurée en suspens, les données recueillies à leur sujet étant alors insuffisantes. Les résultats des évaluations pour chacune des substances peuvent être consultés sur le site internet d'Environnement Canada: Environnement Canada, «Première liste des substances d'intérêt

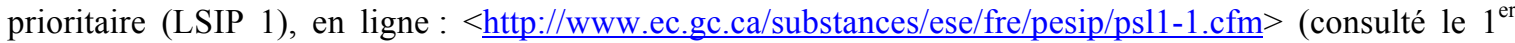
avril 2010). Suite aux recommandations effectuées par un comité consultatif d'experts des ministres, une seconde liste de substances d'intérêt prioritaire a paru en 1995 à la Gazette du Canada. Elle comprenait 25 substances : Environnement Canada, « Deuxième liste des substances d'intérêt prioritaire (LSIP 2) dans le cadre de la Loi canadienne sur la protection de l'environnement » en ligne :
}

$<$ http://www.ec.gc.ca/substances/ese/fre/pesip/psap 4.cfm> (consulté le $1^{\text {er }}$ avril 2010). 
manque d'efficacité. Une telle liste doit toujours être établie sous la L.C.P.E. de 1999, en vertu de l'article $76^{34}$. Toutefois, des modifications importantes sont intervenues avec l'adoption de la L.C.P.E. de 1999. Ainsi, le déclenchement d'une évaluation peut désormais découler de nouvelles considérations et l'évaluation d'une substance existante peut à présent suivre une voie plus succincte. La catégorisation et l'évaluation préalable sont apparues avec la L.C.P.E. de 1999 et se superposent au processus antérieur de liste de substances d'intérêt prioritaire, sans toutefois le remplacer totalement.

Sans entrer ici dans les détails techniques, mentionnons que sept moyens ou canaux peuvent maintenant conduire la Division des substances existantes à procéder à une évaluation des risques :

«(1) la catégorisation des substances figurant sur la liste intérieure des substances (LIS), (2) l'information fournie par l'industrie, (3) les décisions provinciales et internationales visant à interdire ou à restreindre l'usage des substances [en vertu du paragraphe 75(3) de la LCPE (1999)], (4) les suggestions d'inscriptions sur la liste des substances d'intérêt prioritaire soumises par le public, (5) l'évaluation des substances « nouvelles » semblables aux substances existantes [...] (6) Les nouvelles connaissances scientifiques et la surveillance et (7) les évaluations ou la collecte de données, à l'échelle internationale.» ${ }^{35}$

Les substances qui répondent aux critères de catégorisation ${ }^{36}$ seront soumises à une évaluation préalable ${ }^{37}$ par les ministres de l'Environnement et de la Santé, laquelle vise à

\footnotetext{
${ }^{34}$ Environnement Canada, « Liste des substances d'intérêt prioritaire », en ligne :

$<$ http://www.ec.gc.ca/RegistreLCPE/subs list/Priority.cfm> (consulté le 1er avril 2010).

35 Environnement Canada, « Aperçu du Programme des substances existantes à Environnement Canada », en ligne $:<\underline{\text { http://www.ec.gc.ca/substances/ese/fre/overview.cfm }}>$ (consulté le $1^{\text {er }}$ avril 2010).

${ }^{36}$ Environ 4300 substances, dont 200 classées comme hautement prioritaires sont actuellement en phase de collecte d'informations en vertu du Défi lancé par le gouvernement aux entreprises :

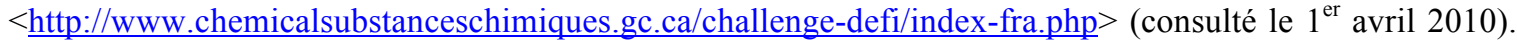
1200 substances peu préoccupantes pour l'environnement ont fait l'objet d'une approche technique d'examen préalable rapide; au terme de cet examen, 754 substances furent considérées non toxiques. Les autres, de même que 2600 substances de priorité moyenne devront faire l'objet de séries successives d'évaluation d'ici 2020. 150 autres substances qui ne sont pas utilisées au Canada mais qui remplissent les critères de la catégorisation ne pourront être commercialisées au Canada avant que les promoteurs ne fournissent les données nécessaires pour qu'elles soient évaluées. Le Plan de gestion des produits chimiques du gouvernement canadien est explicité entre autres dans le document suivant : Gouvernement du Canada, L'examen de la Loi canadienne sur la protection de l'environnement (1999); Réponse provisoire du
} 
déterminer si celles-ci s'avèrent effectivement ou potentiellement toxiques. Suite à cette évaluation, les ministres choisiront parmi les mesures énumérées au paragraphe 77(2) de la L.C.P.E. ${ }^{38}$ celles qu'ils entendent prendre à leur égard. Ces mesures sont soit de ne rien faire, soit d'inscrire la substance sur la liste des substances d'intérêt prioritaire de l'article $76^{39}$, soit de recommander son inscription sur la liste des substances toxiques de l'annexe 1 et de réaliser dans certains cas sa quasi-élimination en vertu des paragraphes 65(3) et 77(4). Par ailleurs, les mesures prévues au paragraphe 77(2) sont aussi applicables à l'endroit des substances qui ont fait l'objet d'un examen par les ministres en vertu du paragraphe 75(3), c'est-à-dire un examen pour déterminer si est effectivement ou potentiellement toxique une substance qui a été interdite explicitement par un gouvernement au Canada ou par un État membre de l'O.C.D.E. ${ }^{40}$ ou qui y a été assujettie à des restrictions importantes pour des raisons environnementales ou de santé. Ces mesures s'appliquent également aux substances inscrites sur la Liste des substances

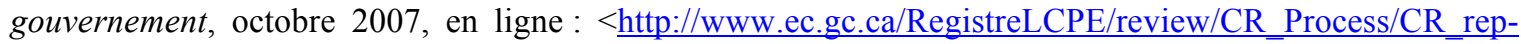
res/CR_rep-res.pdf>, pp. 5 à 7 (consulté le $1^{\text {er }}$ avril 2010).

37 «L'évaluation préalable est la plus élémentaire des évaluations des risques. À cette étape, on évalue les propriétés dangereuses de la substance chimique ainsi que les voies d'exposition à ces dangers pour les humains et l'environnement. La complexité des évaluations préalables peut varier selon le cas» : Gouvernement du Canada, «Qu'est-ce que l'évaluation des risques?», en ligne $<$ http://www.chemicalsubstanceschimiques.gc.ca/about-apropos/assess-eval/what-quoi-fra.php $>$ (consulté le 2 avril 2010).

${ }^{38}$ Art. 74 L.C.P.E.

39 «Les substances chimiques répertoriées dans la Liste des substances d'intérêt prioritaire (LSIP) font l'objet d'une évaluation approfondie, qui peut prendre jusqu'à cinq ans. Les scientifiques gouvernementaux considèrent ces travaux comme hautement prioritaires. L'inscription sur la LSIP peut survenir après une évaluation préalable, après examen d'une décision prise par un autre ordre de gouvernement dans le cadre d'une évaluation, à la demande d'un particulier (sous certaines conditions) ou dans d'autres circonstances» : Gouvernement du Canada, «Qu'est-ce que l'évaluation des risques?», en ligne : $<$ http://www.chemicalsubstanceschimiques.gc.ca/about-apropos/assess-eval/what-quoi-fra.php $>$ (consulté le 2 avril 2010).

${ }^{40}$ Organisation de coopération et de développement économiques, « Sécurité des produits chimiques », en

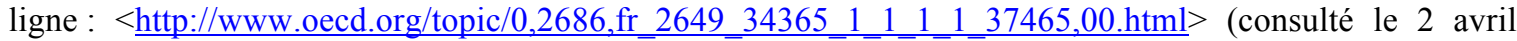
2010). «Le Canada participe activement au Programme sur les produits chimiques de l'OCDE et a établi des liens étroits avec le «Existing Chemicals Program» de l'Agence de protection de l'environnement des États-Unis afin d'échanger de l'information sur les substances préoccupantes » : Environnement Canada, Division des substances existantes, Aperçu du Programme des substances existantes, avril 2007, p. 7. L'OCDE a créé un portail Internet permettant d'accéder directement et gratuitement à de l'information sur les propriétés des substances chimiques ainsi qu'à des évaluations des dangers et des risques : O.E.C.D. «eChemPortal: The Global Portal to Information on Chemical Substances», en ligne : http://webnet3.oecd.org/echemportal/ (consulté le 2 avril 2010). 
d'intérêt prioritaire et qui ont été considérées, après évaluation, potentiellement ou effectivement toxiques ${ }^{41}$.

Des équipes d'Environnement Canada et de Santé Canada cherchent actuellement à se doter d'outils qui leur permettront d'établir des interventions prioritaires quant à l'évaluation des substances catégorisées, tout en continuant l'évaluation des autres substances $^{42}$. La catégorisation complétée entraîne l'évaluation de milliers de substances commerciales et cet exercice, combiné à l'évaluation des autres rejets et substances, s'échelonnera probablement au moins jusqu'en 2020. Le plan canadien de gestion des produits chimiques expose dans ses grandes lignes la marche à suivre pour y parvenir ${ }^{43}$.

\section{La gestion des substances toxiques selon les paramètres prévus à la 'L.C.P.E.'}

\section{Le gouvernement du Canada publiait en 1995 la Politique fédérale de gestion des} substances toxiques qui préconisait la gestion des substances du «berceau au tombeau», soit tout au long de leur cycle de vie et la réalisation de la «quasi-élimination» ${ }^{44}$ de

\footnotetext{
41 Sur le fondement scientifique de l'évaluation environnementale des substances d'intérêt prioritaire; Environnement Canada, « Guide technique », en ligne :

$<$ http://www.ec.gc.ca/substances/ese/fre/pesip/psl2manual.cfm $>$ (consulté le 2 avril 2010). Si après 5 ans, aucune décision n'a été prise au sujet d'une substance inscrite sur la L.S.I.P., quiconque peut déposer auprès du ministre de l'Environnement un avis d'opposition demandant la constitution de la commission de révision prévue à l'article 333 L.C.P.E. (art. 78 (1)).

${ }^{42}$ Voir à titre d'exemple : Gouvernement du Canada, « Projet pilote pour l'évaluation préalable », en

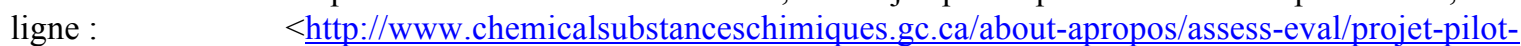
project/index-fra.php $>$ (consulté le 2 avril 2010).
}

43 Gouvernement du Canada, «Plan de gestion des produits chimiques», en ligne :

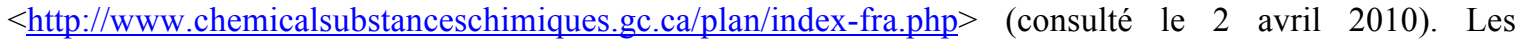
évaluations préalables des 200 substances hautement prioritaires identifiées par l'exercice de catégorisation sont en bonne voie d'être complétées. En mars 2010, le gouvernement publiait les évaluations préalables finales des substances du septième lot. Sur ce processus et pour consulter les évaluations préalables complétées, voir : Gouvernement du Canada, «Substances chimiques du septième lot du Défi», en ligne :

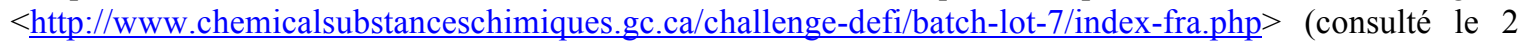
avril 2010).

44 La quasi-élimination, également désignée sous l'expression «élimination virtuelle », est définie aux articles 65 et 65.1 de la L.C.P.E. Il s'agit de la «réduction de la libération dans l'environnement des substances toxiques les plus dangereuses à une concentration à laquelle on ne peut plus les mesurer exactement»: Comité sénatorial permanent de l'énergie, de l'environnement et des ressources naturelles, Loi canadienne sur la protection de l'environnement (1999, ch. 33); Rx: Renforcez et appliquez avec soin, mars 2008, Deuxième session de la trente-neuvième législature, en ligne : <http://www.parl.gc.ca/39/2/parlbus/commbus/senate/com-f/enrg-f/rep-f/rep06mar08-f.htm>, $\quad$ p. 17 (consulté le 3 avril 2010). La quasi-élimination nécessite donc d'établir une «limite de dosage», soit «la 
certaines substances, soit celles qui étaient persistantes ou bioaccumulables et qui résultaient de l'activité humaine. C'est dans cette ligne de pensée que s'inscrivent toujours aujourd'hui les objectifs gouvernementaux de gestion des substances jugées toxiques. Le préambule de la L.C.P.E. marque d'ailleurs clairement cette intention :

«Attendu que le gouvernement du Canada $[\ldots]$ reconnaît la nécessité de procéder à la quasi-élimination des substances toxiques les plus persistantes et bioaccumulables et de limiter et gérer les polluants et déchets dont le rejet dans l'environnement ne peut être évité;

qu'il s'efforcera d'éliminer les menaces à la diversité biologique au moyen de la prévention de la pollution, de la réglementation et de la gestion des risques d'effets nocifs de l'utilisation et du rejet de substances toxiques, de polluants et de déchets et de la quasi-élimination des substances toxiques persistantes et bioaccumulables.»

À titre de mesure obligatoire, les ministres doivent proposer, s'il est déterminé qu'une substance est effectivement ou potentiellement toxique et s'ils sont convaincus en se fondant sur l'évaluation préalable, qu'elle «peut avoir à long terme un effet nocif sur l'environnement, qu'elle est persistante et bioaccumulable au sens des règlements et qu'elle présente, d'après des études en laboratoire ou autres, une toxicité intrinsèque pour les êtres humains ou les organismes autres que les organismes humains et que sa présence dans l'environnement est du principalement à l'activité humaine» son inscription à la liste des substances toxiques de l'Annexe I de la Loi, en vertu du paragraphe 77(3) L.C.P.E. Les ministres doivent également proposer la réalisation de la quasi-élimination d'une substance, lorsqu'ils sont convaincus qu'elle est persistante, bioaccumulable, présente dans l'environnement principalement en raison de l'activité humaine et qu'elle n'est pas une substance inorganique, ou un radionucléide, d'origine naturelle, s'ils proposent aussi son inscription à l'annexe $1^{45}$. Par ailleurs, selon le paragraphe 90(1), «[s]’il est convaincu qu'une substance est toxique, le gouverneur en conseil peut prendre, sur recommandation des ministres, un décret d'inscription de la substance sur la liste de l'annexe 1». Lorsqu'elles ont été inscrites par le gouvernement sur la liste des substances toxiques, les

concentration la plus faible d'une substance qui peut être mesurée avec exactitude au moyen de méthodes d'analyse et d'échantillonnage précises mais courantes» : art. 65.1 L.C.P.E.

${ }^{45}$ Paragraphe 77(4) L.C.P.E. 
substances peuvent dès lors faire l'objet d'un règlement du gouverneur en conseil, notamment en ce qui a trait à leur utilisation, leur fabrication, les conditions de vente, d'importation et d'exportation, etc. ${ }^{46}$ À titre d'exemple, six gaz à effet de serre ont été inscrits à l'annexe 1 , en novembre $2005^{47}$. Ils répondaient selon le gouvernement au critère b) de la définition de substance toxique au sens de l'article 64 de la Loi, puisqu'il y avait lieu de croire qu'ils constituaient ou pouvaient constituer un danger pour l'environnement essentiel à la vie.

Dans les deux ans qui suivent la publication d'une déclaration précisant que la mesure à l'endroit d'une substance consiste à recommander son inscription sur la liste de l'annexe 1, le ministre de l'Environnement doit publier un projet de texte - règlement ou autre portant sur les mesures de prévention ou de contrôle relatives à cette substance ${ }^{48}$. Ainsi, en vertu des dispositions de la L.C.P.E., le ministre de l'Environnement dispose d'une période de vingt-quatre mois pour proposer un instrument de contrôle d'une substance, et d'une période de dix-huit mois supplémentaires pour compléter cet instrument après consultation $^{49}$. Les instruments de contrôle mentionnés dans la L.C.P.E. sont les règlements, les codes de pratique, les plans d'urgence, les plans de prévention de la pollution, les directives ou les ententes d'équivalence avec les provinces. Soulignons que l'obligation législative consiste à exiger du ministre qu'il adopte «des mesures de prévention ou contrôle» à l'intérieur du délai fixé par la Loi, ce qui n'empêche donc pas que des mesures non prévues à la L.C.P.E. soient éventuellement prises de façon complémentaire pour assurer le contrôle de la substance, telles des ententes de performance environnementale entre le gouvernement et l'industrie.

En somme pour chaque substance considérée toxique au sens de la Loi, une stratégie de gestion de risques sera donc élaborée par une équipe du ministère de l'Environnement chargée de répertorier les secteurs industriels qui l'utilisent, les quantités rejetées, les acteurs impliqués, de même que les considérations socio-économiques qui pourront

\footnotetext{
${ }^{46}$ Art. 93 L.C.P.E.

${ }^{47}$ Ces inscriptions ont cependant fait l'objet d'une controverse, puisque certains considèrent que les gaz à effet de serre ne correspondent pas à ce qui doit être entendu de l'expression «substances toxiques ».

${ }^{48}$ Par. 91(1) L.C.P.E.

${ }^{49}$ Art. 91 et 92 L.C.P.E.
} 
influer sur le choix des instruments de gestion mis en place. La stratégie mise de l'avant doit couvrir toutes les sources de rejet de la substance et tous les secteurs d'importance de production et d'utilisation de celle-ci en plus de s'intéresser à tout son cycle de vie. En outre, la stratégie fera l'objet d'une consultation publique avant que ne soient développés les instruments de gestion qu'elle propose. Ensuite, la même équipe tentera d'identifier, selon le secteur d'activités, le ou les outils les plus prometteurs pour assurer la gestion des risques associés à la substance. Une étude des coûts / bénéfices sera souvent effectuée pour bien comprendre les impacts des mesures envisagées tant au niveau des secteurs industriels que du gouvernement et du grand public ${ }^{50}$.

Après cet exercice, la stratégie de gestion des risques est présentée au sous-ministre. Avant sa divulgation publique, elle fera aussi l'objet de discussions devant le Comité consultatif national. Ce comité comprend des représentants des gouvernements provinciaux et autochtones et est entre autres chargé de conseiller le ministre de l'Environnement, selon les termes de l'al. 6(1)b) de la L.C.P.E., "sur un cadre intergouvernemental d'action concertée pour la gestion des substances toxiques» ${ }^{51}$. La stratégie sera enfin présentée pour consultation auprès du public, les industries et les groupes environnementaux en particulier.

Alors que dans le contexte de l'évaluation des risques posés par les substances, les critères à considérer sont d'ordre strictement scientifique, il en va autrement lorsque arrive le moment de décider des mesures de contrôle de la pollution devant être prises à l'endroit

\footnotetext{
50 «En général, quand on décide s'il convient d'agir pour gérer un risque, l'analyse coûts-avantages implique la détermination et la pondération des impacts réels et potentiels de la décision. Toutefois, dans le contexte du principe de la prudence, l'incertitude scientifique et l'information limitée concernant les rapports complexes peuvent empêcher une analyse coûts-avantages détaillée ou officielle. De plus, les situations entraînant des dommages graves ou irréversibles potentiels sont souvent imprévues et exigent une intervention urgente en fonction des connaissances scientifiques et socio-économiques disponibles. Ces facteurs peuvent limiter la faisabilité d'une analyse coûts-avantages exhaustive ou officielle»: Environnement Canada / Santé Canada, Document d'orientation concernant la Loi canadienne sur la protection de l'environnement (1999) et le principe de prudence : amélioration de la prise de décisions dans l'incertitude, pp.12-13.

51 Il arrive que cette consultation mène à la conclusion qu'une substance considérée toxique est adéquatement gérée au niveau provincial et qu'elle ne commande donc pas au niveau national d'actions supplémentaires. Dans ces cas, des ententes administratives ou d'équivalence sont conclues avec les provinces en vertu desquelles celles-ci assurent la gestion de la substance, sous réserve d'en rendre périodiquement compte au ministre de l'Environnement fédéral. Celui-ci demeure tout de même
} 
d'une substance considérée toxique. Le paragraphe 2 (1.1) L.C.P.E. mentionne dans ses alinéas a), b) et c) les facteurs qui doivent être pris en compte par le gouvernement du Canada avant de prendre des mesures conformément à l'alinéa (1) a. $1^{52}$ :

«a) les avantages humains et écologiques découlant, à court et à long terme, de la mesure de protection de l'environnement;

b) les conséquences économiques positives découlant de la mesure, notamment les économies découlant des progrès et innovations en matière de technologie, de santé et d'environnement;

c) tout autre avantage découlant de la mesure.»»

Le paragraphe 91(5) mentionne quant à lui divers facteurs que les ministres peuvent prendre en considération pour décider des mesures de prévention ou de contrôle à instaurer à l'égard des substances effectivement ou potentiellement toxiques: «les renseignements contenus dans les plans visés à l'article 79», c'est-à-dire les plans requis pour la quasi-élimination, «les risques d'atteinte à l'environnement ou à la santé qui sont liés à la substance et signalés dans le résumé publié au titre du paragraphe 77(6) ainsi que toute autre question d'ordre social, économique ou technique». Compte tenu de cette énumération, nous pouvons anticiper que le principe de précaution a un rôle à jouer en vertu de la Loi dans la prise de décision du gouvernement.

\section{L'INCERTITUDE SCIENTIFIQUE ET SA PRISE EN COMPTE JURIDIQUE DANS LE CONTEXTE DE LA 'L.C.P.E.'}

La communauté internationale a reconnu la nécessité de réaliser une meilleure médiation entre les bienfaits des avancées technologiques et de la mondialisation, d'un côté, et les risques inconnus qu'apportent ces changements rapides, de l'autre. Compte tenu des incertitudes scientifiques entourant la détermination de la toxicité potentielle des produits

responsable de la gestion de la substance toxique devant le Parlement et il doit pouvoir informer adéquatement les citoyens à ce sujet.

52 Cet alinéa indique que le gouvernement doit «prendre des mesures préventives et correctives pour protéger, valoriser et rétablir l'environnement». 
chimiques et considérant également la sensibilité accrue du public face aux risques sanitaires et environnementaux, le principe de précaution a occupé une place grandissante sur la scène publique au cours des dernières années. Le principe a été énoncé dans plusieurs traités et déclarations internationaux avant son introduction à la L.C.P.E., parmi lesquels la Déclaration de Rio de 1995 figure au premier plan $^{53}$. D'ailleurs, certaines discussions ont également eu lieu au Canada au sujet de l'application du principe à divers enjeux, le plus souvent en matière de santé et de protection de l'environnement. Ainsi, des travaux de la Commission mixte internationale sur les Grands Lacs ont porté sur l'application au domaine des substances toxiques du principe de précaution ainsi que sur les notions de persistance, de bioaccumulation, de poids de la preuve et de quasiélimination, qui y sont fortement liées ${ }^{54}$. Les membres de la Commission ont entre autres insisté sur l'importance du principe de précaution eu égard à la persistance et au potentiel de bioaccumulation des substances chimiques, une interdiction complète d'une substance bioaccumulable étant préconisée lorsqu'une forte probabilité de lien de causalité était établie entre le danger qu'elle représentait et ses effets potentiels. Ces travaux ont certainement influencé en partie le législateur canadien au moment de rédiger la L.C.P.E. de 1999, bien que de nombreux autres éléments confèrent au principe de précaution un sens distinct dans le cadre de cette Loi.

La révision de la L.C.P.E. de 1988 a ainsi conduit le législateur à favoriser une approche de prudence accrue en matière d'évaluation et de gestion des substances toxiques au

\footnotetext{
${ }^{53}$ Le Principe 15 de la Déclaration de Rio sur l'environnement et le développement se lit comme suit : «Pour protéger l'environnement, des mesures de précaution doivent être largement appliquées par les États selon leurs capacités. En cas de risque de dommages graves ou irréversibles, l'absence de certitude scientifique absolue ne doit pas servir de prétexte pour remettre à plus tard l'adoption de mesures effectives visant à prévenir la dégradation de l'environnement.» Pour un historique du principe de précaution; voir P. Kourilsky et G. Viney, Le principe de précaution, Paris, Éditions Odile Jacob, 2000, pp. 253 et ss.; voir aussi $\mathrm{H}$. Trudeau, «Du droit international au droit interne ; l'émergence du principe de précaution en droit de l'environnement», (2003) 28 Queen's Law Journal 455.

${ }^{54}$ En particulier : Commission mixte internationale, Septième rapport biennal en vertu de l'Accord de 1978 relatif à la qualité de l'eau dans les Grands Lacs présenté aux Gouvernements des États-Unis et du Canada et aux Gouvernements des États et de la Province du Bassin des Grands Lacs, 1994, en ligne : < http://www.ijc.org/php/publications/html/7brf.html> consulté le 9 avril 2010; Groupe de travail sur l'élimination virtuelle, A Strategy for Virtual Elimination of Persistent Toxic Substances (Volumes 1 et 2), Rapport final présenté à la Commission mixte internationale, Windsor, Ontario, août 1993 et T. Williams, «Élimination virtuelle de la pollution résultant du rejet des substances toxiques», 26 juillet 2006, Bibliothèque du Parlement, Canada, en ligne :
} 
Canada, notamment en fixant des délais plus courts pour procéder à l'évaluation et la gestion des risques, en instituant le processus de catégorisation, en établissant certaines obligations concernant la quasi-élimination de substances persistantes et bioaccumulables, et en rendant déterminantes les décisions d'autres instances gouvernementales, provinciales ou d'États membres de l'O.C.D.E. quant à la déclaration de toxicité des substances $^{55}$. L'un des documents préparatifs commandés dans le cadre de cette révision législative portait spécifiquement sur le principe de précaution ${ }^{56}$.

Ainsi, le principe de précaution a été intégré à la L.C.P.E. de 1999 pour donner une orientation à la prise en compte juridique des situations d'incertitude scientifique, particulièrement au stade de l'évaluation des risques. Nous l'étudierons dans la première sous-section (2.1). Également, la méthode du poids de la preuve, certes moins connue des juristes, doit être expliquée car elle concerne également l'analyse des données scientifiques dans l'évaluation des risques et est mentionnée à la L.C.P.E. Elle fera l'objet de courts développements dans la seconde sous-section (2.2).

\section{Le principe de précaution}

Dans la L.C.P.E. de 1999, le principe de précaution est énoncé au préambule ainsi qu'aux articles 2 (1), 6 (1.1) et 76.1, qui concernent respectivement l'application administrative de la Loi, le comité consultatif national et l'évaluation des substances chimiques ${ }^{57}$. Ce principe est toutefois désigné dans la version française de la L.C.P.E. sous l'expression

\footnotetext{
$<$ http://www2.parl.gc.ca/content/lop/researchpublications/prb0626-f.htm> (consulté le 8 avril 2010).

55 R. Daigneault, «La L.C.P.E. 1999 : portée et enjeux», Formation permanente, Barreau du Québec, Développements récents en droit de l'environnement (2000), Cowansville, Éditions Yvon Blais, 2000, p. 223; Chambre des communes du Canada, Comité permanent de l'environnement et du développement durable, Notre santé en dépend! Vers la prévention de la pollution : Examen de la LCPE, Ottawa, Service des publications, 1995. Gouvernement du Canada, Mesures législatives sur la protection de l'environnement conçues pour l'avenir - Une LCPE renouvelée, Examen de la LCPE: Réponse du gouvernement, Ottawa, Ministre des Approvisionnements et Services, 1995.

56 D. VanderZwaag, La LCPE et le principe ou l'approche précaution, Examen de la LCPE: Document d'élaboration des enjeux 18, Canada, Ministre des Approvisionnement et Services, 1994.

${ }^{57}$ Environnement Canada, «Principes directeurs de la LCPE (1999) et autres politiques», en ligne :

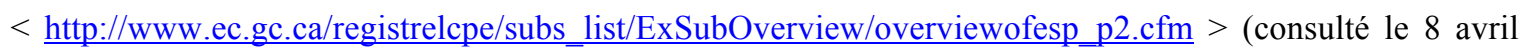
2010); H. Trudeau, «Du droit international au droit interne ; l'émergence du principe de précaution en droit de l'environnement», (2003) 28 Queen's Law Journal 455, pp. 479 à 482.
} 
«principe de prudence» ${ }^{58}$. Bien que sa désignation puisse varier selon les documents dans lesquels il apparaît, le principe de précaution concerne toujours la prise de décision dans un contexte d'incertitude scientifique en matière de gestion de risques. Sa formulation comprend habituellement trois axes: la nécessité de prendre action, l'existence d'un risque de préjudice grave ou irréversible et l'absence de certitude absolue. Les paramètres plus précis de son application peuvent être prévus dans les textes juridiques dans lesquels il est formulé. Certains instruments se réfèrent ainsi à «l'absence de lien de causalité» plutôt qu'à «l'absence de certitude scientifique absolue» ${ }^{59}$, d'autres à des «dommages sévères» plutôt qu'à des «dommages graves ou irréversibles», d'autres encore insistent moins ou davantage sur la prise en considération de facteurs socio-économiques au moment de l'adoption de mesures visant à prévenir la dégradation de l'environnement ${ }^{60}$.

La formulation du principe de précaution choisie par le législateur canadien dans la L.C.P.E. présente beaucoup de similarités avec celle prévue à la Déclaration de Rio ${ }^{61}$. L'alinéa 2(1)a) de la L.C.P.E. précise que pour l'exécution de la Loi :

«[...], le gouvernement fédéral doit, compte tenu de la Constitution et des lois du Canada et sous réserve du paragraphe $(1.1): a$ ) exercer ses pouvoirs de manière à protéger l'environnement et la santé humaine, à appliquer le principe de la prudence, si bien qu'en cas de risques de dommages graves ou irréversibles à

\footnotetext{
${ }^{58}$ En version anglaise «precautionary principle». Nous avons résolu d'utiliser dans le présent texte l'expression «principe de précaution».

${ }^{59}$ Notons toutefois qu'il n'est rien de tel que la certitude scientifique absolue. Partant de ce fait, il est clair que c'est plutôt en termes de "degré d'incertitude » ou de " degré de certitude » que doit réellement être défini et appliqué le principe de précaution.

${ }^{60}$ De nombreux ouvrages doctrinaux sont consacrés au principe de précaution. Pour des développements additionnels sur ces questions, voir entre autres : N. de Sadeleer, Les principes du pollueur-payeur, de prévention et de précaution, Bruxelles, Bruylant, 1999; J. Cameron et J. Abouchar, «The Precautionary Principle : A Fundamental Principle of Law and Policy for the Protection of the Global Environment» (1991) 14 Boston College International \& Comparative Law Review 1; J. Cameron, «Future Directions in International Environmental Law : Precaution, Integration and Non-state Actors » (1996) 19 Dalhousie Law Journal 122; E. Hey, "The Precautionary Concept in Environmental Policy and Law : Institutionalizing Caution» (1992) 4 The Georgetown Int'l Envtl. Law Review 303; J. E. Hickey et V. R. Walker, « Refining the Precautionary Principle in Environmental Law » (1995) 14 Virginia Environmental Law Journal 423; O.McIntyre et T. Mosedale, "The Precautionary Principle as a Norm of Customary International Law » (1997) 9 Journal of Environmental Law 221; D. Freestone et E. Hey, eds, The Precautionary Principle and International Law. The Challenge of Implementation, The Hague, London, Boston, Kluwer International Law, 1996.

${ }^{61}$ Supra, note 52.
} 
l'environnement, l'absence de certitude scientifique absolue ne doit pas servir de prétexte pour remettre à plus tard l'adoption de mesures effectives visant à prévenir la dégradation de l'environnement, ainsi qu'à promouvoir et affermir les méthodes applicables de prévention de la pollution.»

L'article 76.1 de la L.C.P.E., intégré à la Partie 5 de la Loi qui met en place le régime législatif visant l'évaluation et la gestion des substances toxiques, se lit comme suit : «Les ministres appliquent la méthode du poids de la preuve et le principe de la prudence lorsqu'ils procèdent à l'évaluation et aux examens ci-après mentionnés et à l'évaluation de leurs résultats :

a) l'évaluation préalable en vertu de l'article 74;

b) l'examen, en vertu du paragraphe 75(3), de la décision d'une autre instance qui, de leur avis, est, à la fois, fondée sur des considérations scientifiques et pertinente pour le Canada;

c) l'examen afin de déterminer si une substance inscrite sur la liste des substances d'intérêt prioritaire est effectivement ou potentiellement toxique.»

Le principe de précaution, de même que ses paramètres d'application, ne sont pas davantage précisés dans la L.C.P.E. Il apparaît cependant certain que dans la prise de décision à l'endroit des substances toxiques, le gouvernement fédéral se doit de respecter le principe, tant au stade de l'évaluation des risques qu'à celui de leur gestion. L'article 76.1 vise ainsi clairement l'étape de l'évaluation des risques, tandis que l'article 2 (1) est entre autres applicable à la gestion des risques ${ }^{62}$.

Pour avoir une idée plus complète de ce que peut signifier le principe de précaution dans l'exercice du pouvoir décisionnel au sein du gouvernement canadien, il faut se référer à certains documents qui explicitent son application. Le gouvernement du Canada, par la voix du Bureau du Conseil privé, a publié en 2003 un document intitulé Cadre d'application de la précaution dans un processus décisionnel scientifique en gestion du

\footnotetext{
${ }^{62}$ Dans le contexte de la gestion des risques cependant, les décideurs publics seront plus à même de faire intervenir des considérations économiques dans le choix de mesures pour contrer les risques, ce que permettent d'ailleurs les dispositions de la Loi : voir nos remarques, supra, p. 17. Voir aussi : H. Trudeau, «Du droit international au droit interne; l'émergence du principe de précaution en droit de l'environnement», (2003) 28 Queen's Law Journal 455, pp. 491 à 495.
} 
risque $^{63}$. Ce document vise notamment à clarifier la position canadienne face à «l'application de la précaution dans la prise de décisions scientifiques dans les secteurs d'activité réglementés au palier fédéral ${ }^{64}$ et à uniformiser cette application parmi les différents ministères fédéraux. Le gouvernement a ainsi proposé 10 principes directeurs visant à traduire globalement la perspective canadienne en la matière :

«Cinq principes généraux d'application

1. La précaution est une démarche légitime et particulière de décision dans la gestion du risque.

2. Il est légitime que les décisions soient guidées par le niveau de protection contre le risque que choisit la société.

3. L'application de la précaution doit reposer sur des données scientifiques solides et sur leur évaluation; la nature des données scientifiques et la partie chargée de les produire peuvent changer avec l'évolution du savoir.

4. Il devrait y avoir des mécanismes pour réévaluer le fondement des décisions et pour tenir éventuellement d'autres consultations dans un processus transparent.

5. Il convient d'assurer un degré élevé de transparence, de reddition de comptes et de participation significative du public.

Cinq principes d'application des mesures de précaution

6. Les mesures de précaution devraient être sujettes à réexamen selon l'évolution de la science, de la technologie et du niveau de protection choisi par la société.

7. Les mesures de précaution devraient être proportionnelles à la gravité possible du risque que l'on veut gérer et au niveau de protection choisi par la société.

8. Les mesures de précaution devraient être non discriminatoires et concorder avec celles prises dans des circonstances similaires.

\footnotetext{
${ }^{63}$ Gouvernement du Canada, Bureau du Conseil privé, Cadre d'application de la précaution dans un processus décisionnel scientifique en gestion du risque, 2003, en ligne: < bcp.gc.ca/index.asp?lang=fra\&page $=$ information $\&$ sub $=$ publications $\&$ doc $=$ precaution/precaution f.htm $>$ (consulté le 8 avril 2010).

${ }^{64} I d .$, p. 2.
} 
9. Les mesures de précaution devraient être efficientes et avoir pour objectif d'assurer (i) un avantage net global à la société au moindre coût et (ii) un choix judicieux de mesures.

10. Si plusieurs options réunissent ces caractéristiques, on devrait choisir celle qui entrave le moins le commerce.» ${ }^{65}$

Alors que le Principe 2 du Cadre énonce qu' «il est légitime que les décisions soient guidées par le niveau de protection contre le risque que choisit la société», le Principe 3 agit à titre de tempérament en précisant que «l'application de la précaution doit reposer sur des données scientifiques solides et sur leur évaluation; la nature des données scientifiques et la partie chargée de les produire peuvent changer avec l'évolution du savoir.»

L'ensemble de ces principes constitue le point de vue fédéral s'appliquant, sauf exceptions, aux situations d'évaluation et de gestion du risque. Cependant, il importe de rappeler que le principe de précaution est employé différemment selon les circonstances et les facteurs particuliers de chaque cas ${ }^{66}$.

En complément au Cadre fédéral applicable globalement au Canada, un document interne d'orientation a également été élaboré par Environnement Canada et Santé Canada afin de préciser l'interprétation du principe de précaution dans le cadre de la L.C.P.E ${ }^{67}$. Intitulé Document d'orientation concernant la Loi canadienne sur la protection de l'environnement (1999) et le principe de prudence, il présente des balises additionnelles, expliquant ce qui devrait généralement être entendu du principe dans le cadre de la Loi et guidant la façon dont il doit être appliqué, particulièrement en matière de gestion et d'évaluation des substances chimiques. Ce document, destiné à harmoniser l'application du principe de prudence au sein des diverses divisions concernées, résume comme suit le spectre des considérations soulevées par le principe :

\footnotetext{
${ }^{65} I d$., pp. 6 et ss.

${ }^{66}$ Id., p. 6.

${ }^{67}$ Environnement Canada / Santé Canada, Document d'orientation concernant la Loi canadienne sur la protection de l'environnement (1999) et le principe de prudence-Amélioration de la prise de décisions dans l'incertitude.
} 
«Le principe de prudence s'inscrit dans un continuum qui tient compte, cas par cas : du degré et du type de dommage présumé, compte tenu de sa gravité ou de son irréversibilité potentielle ainsi que de sa nature et de sa distribution possibles, soit la «menace», et du degré d'incertitude lié aux dommages potentiels. S'il existe une base pour établir une présomption scientifique de dommages graves ou irréversibles, le niveau précis de certitude scientifique requis pour justifier des mesures préventives en vertu de la LCPE (1999) variera selon les circonstances. En règle générale, plus la menace est grave ou potentiellement irréversible, moins il faut compter sur une certitude scientifique absolue pour justifier la prise de mesures préventives.

Lorsque la menace est grave ou irréversible, une prépondérance ou un équilibre de preuves peut suffire à justifier une forme ou une autre de mesure préventive. Il peut convenir d'agir en se fondant uniquement sur une indication initiale de dommages potentiels $[\ldots] .{ }^{68}$

De plus, ce document souligne que l'application efficace du principe de la prudence repose sur la qualité des pratiques scientifiques et d'évaluation des risques, ainsi que sur la communication en temps opportun des résultats de recherche aux décideurs ${ }^{69}$.

Les guides Aperçu du Programme des substances existantes et Aperçu de l'évaluation écologique des substances en vertu de la Loi canadienne sur la protection de l'environnement (1999) fournissent quant à eux des renseignements plus précis quant au processus suivi par la Division des substances existantes à l'étape de l'évaluation ainsi que les paramètres étudiés en vue de déterminer le statut des substances candidates. Le premier résume en ces mots le processus de prise de décision en gestion de risques :

«Toutes les évaluations comportent des éléments d'incertitude, cela indépendamment de leur degré de détail ou de leur caractère exhaustif. L'incertitude peut influencer l'estimation de l'importance et de la probabilité d'un risque et avoir un effet sur la conclusion de l'évaluation. La détermination et la communication ouverte des incertitudes et des mesures prises pour les prendre en compte sont donc essentielles à l'élaboration de conclusions fiables. Cela

\footnotetext{
${ }^{68}$ Id., pp. 16-17.

${ }^{69} I d$., pp. 13 à 16.
} 
comprend la détermination des sources individuelles d'incertitude et l'examen de leurs effets cumulatifs sur le niveau de confiance de la conclusion de l'évaluation.

Il arrive que le manque de renseignements empêche de perfectionner les scénarios d'évaluation, ce qui donne lieu à des conclusions fondées sur des hypothèses par défaut prudentes. Il est alors offert aux intervenants de fournir des renseignements permettant de réduire l'incertitude. En l'absence de ces renseignements, les décisions d'appliquer ou non des mesures de prévention ou de contrôle ne peuvent être prises qu'en mettant en regard le potentiel de risque et l'ampleur des effets tout en connaissant la nature des incertitudes. ${ }^{70}$

Le second guide, Aperçu de l'évaluation écologique des substances en vertu de la Loi canadienne sur la protection de l'environnement (1999), illustre quant à lui les étapes de l'évaluation écologique, énumère le type de données étudiées, explique la façon dont sont calculées les valeurs qui mènent au verdict scientifique quant aux substances et décrit brièvement comment est prise en compte l'incertitude. L'un des éléments soulignés dans ce guide est celui selon lequel la prise en compte de l'incertitude se traduit par l'application de facteurs d'évaluation décuplés au moment de calculer la concentration estimée sans effet (c'est-à-dire, les seuils de rejets acceptables) ${ }^{71}$.

Au cours des dernières années, les ressources fédérales dans le domaine des substances chimiques ont largement été mobilisées autour de la complétion de la catégorisation exigée par la Loi, de sorte que l'article 76.1 de la L.C.P.E., sur le principe de prudence et la méthode du poids de la preuve au stade de l'évaluation préalable, a peu été appliqué à ce jour. D'ailleurs, très peu de documents d'interprétation, de critères ou d'indications quant à la manière dont doivent être comprises les dispositions sur le principe de précaution dans le cadre du Programme des substances existantes n'étaient disponibles jusqu'à la fin de l'exercice de catégorisation. Ces documents laissent encore plusieurs questions en suspens. Des explications additionnelles permettraient d'accroître la

\footnotetext{
70 Environnement Canada, Division des substances existantes, Aperçu du Programme des substances existantes, Canada, avril 2007, p. 11 et 12.

${ }^{71}$ Environnement Canada, Aperçu de l'évaluation écologique des substances en vertu de la Loi canadienne sur la protection de l'environnement (1999), Canada, juin 2007, p. 20.
} 
prévisibilité des décisions prises à l'égard des substances chimiques susceptibles d'être toxiques.

\section{La méthode du poids de la preuve}

Rappelons ici que l'article 76.1 de la L.C.P.E. se lit comme suit :

Les ministres appliquent la méthode du poids de la preuve et le principe de la prudence lorsqu'ils procèdent à l'évaluation et aux examens ci-après mentionnés et à l'évaluation de leurs résultats :

a) l'évaluation préalable en vertu de l'article 74 ;

b) l'examen, en vertu du paragraphe 75(3), de la décision d'une autre instance qui, de leur avis, est, à la fois, fondée sur des considérations scientifiques et pertinente pour le Canada;

c) l'examen afin de déterminer si une substance inscrite sur la liste des substances d'intérêt prioritaire est effectivement ou potentiellement toxique.

La méthode du poids de la preuve n'est pas définie par la L.C.P.E. ${ }^{72}$ Néanmoins, selon le Document d'orientation concernant la Loi canadienne sur la protection de l'environnement (1999) et le principe de prudence, la méthode du poids de la preuve « $[\ldots]$ consiste en une évaluation pondérée de toutes les sources de données sur les risques et tient compte du niveau d'incertitude scientifique. On pourrait accorder plus d'importance à une source de données pour déterminer les risques en fonction de chaque

\footnotetext{
${ }^{72}$ Sur la méthode du poids de la preuve, des explications additionnelles peuvent être trouvées dans les documents suivants : Commission mixte internationale, Rapports biennaux en vertu de l'Accord de 1978 relatif à la qualité de l'eau dans les Grands Lacs présenté aux Gouvernements des États-Unis et du Canada et aux Gouvernements des États et de la Province du Bassin des Grands Lacs, les 6, 7 et 8 rapports en particulier, 1992, 1994 et 1996, en ligne : <http://www.ijc.org/fr/publications/rpts bi.htm> consulté le 9 avril 2010; Commission mixte internationale, Conseil consultatif scientifique des Grands Lacs, «1993-1995 Priorities and Progress Under the Great Lakes Water Quality Agreement, Chapter Two : Great Lakes Science Advisory Board», en ligne : < http://www.ijc.org/rel/boards/sab/pr9395.html >, (consulté le 9 avril 2010); Chambre des communes du Canada, Comité permanent de l'environnement et du développement durable, Notre santé en dépend! Vers la prévention de la pollution : Examen de la LCPE, Ottawa, Service des publications, 1995. Gouvernement du Canada, Mesures législatives sur la protection de l'environnement conçues pour l'avenir - Une LCPE renouvelée, Examen de la LCPE : Réponse du gouvernement, Ottawa, Ministre des Approvisionnements et Services, 1995.
} 
cas. Le résultat de l'évaluation scientifique et la méthode du poids de la preuve permettent de décider si une substance est toxique»» ${ }^{73}$.

Pour sa part, le guide Aperçu de l'évaluation écologique des substances en vertu de la Loi canadienne sur la protection de l'environnement (1999) décrit ainsi cette méthode:

«La méthode du poids de la preuve comporte l'utilisation de plusieurs éléments d'information constitutifs afin de prendre des décisions dans toutes les étapes de l'évaluation, dont la caractérisation des risques. Ces éléments comprennent notamment les quotients de risque, les résultats des évaluations probabilistes, la preuve de dommages causés sur le terrain, la preuve de la persistance et le potentiel de bioaccumulation ainsi que les niveaux d'exposition élevés ou croissants. L'utilisation de toutes les preuves disponibles permet de mieux caractériser les incertitudes et peut contribuer à réduire les incertitudes globales liées aux décisions. Plutôt que d'avoir confiance en une seule méthode d'évaluation écologique, on examine des éléments d'information distincts qu'on regroupe logiquement et dont on se sert pour tirer des conclusions. La méthode du poids de la preuve a pour but d'accroître la rigueur scientifique de l'évaluation écologique.» ${ }^{74}$

La qualité et la fiabilité des données scientifiques disponibles déterminent pour une large part la décision prise au sujet d'une substance. L'information recueillie peut prendre diverses formes: données expérimentales, données sur des analogues étroitement apparentés, prédictions des modèles, jugement expert... Toutes les données de qualité et les estimations jugées exactes des modèles seront prises en considération au moment d'appliquer la méthode du poids de la preuve. Pour l'évaluation d'un paramètre en particulier, on préférera cependant certaines formes de données par rapport à d'autres. Par exemple, les données expérimentales primeront généralement sur les données prédites par des modèles ${ }^{75}$. Le gouvernement a recueilli pour certaines substances des ensembles de

\footnotetext{
73 Environnement Canada / Santé Canada, Document d'orientation concernant la Loi canadienne sur la protection de l'environnement (1999) et le principe de prudence - Amélioration de la prise de décisions dans l'incertitude, p. 6.

${ }^{74}$ Environnement Canada, Aperçu de l'évaluation écologique des substances en vertu de la Loi canadienne sur la protection de l'environnement (1999), Canada, juin 2007, pp. 6-7.

${ }^{75}$ Id., p. 11.
} 
données détaillées fondées sur des données expérimentales. Il demeure cependant que, pour la majorité des substances, ces données sont très limitées ou inexistantes ${ }^{76}$.

\section{LES PARAMÈTRES D'APPLICATION DU PRINCIPE DE PRÉCAUTION DANS L'ÉVALUATION ET LA GESTION DES SUBSTANCES TOXIQUES : QUELQUES PISTES DE RÉFLEXION}

En vertu de l'article 343 de la L.C.P.E. de 1999, le Parlement devait procéder à un examen de la Loi cinq ans après son entrée en vigueur. Pourtant ce n'est qu'en avril 2007, que le Comité permanent de l'environnement et du développement durable (CPEDD) a présenté son rapport sur l'examen de la Loi à la Chambre des communes ${ }^{77}$. Une réponse provisoire du gouvernement a été formulée pour y donner suite ${ }^{78}$. Prenant en considération les consultations publiques tenues dans le processus d'examen de la loi et les recommandations émises, le gouvernement fédéral estime inutile et improductif d'envisager une réforme en profondeur de la L.C.P.E à ce stade-ci, mais considère toutefois que «[...] l'apport de changements assez modestes pourrait améliorer la mise en œuvre des programmes et renforcer la capacité du gouvernement à atteindre ses objectifs en matière de protection de l'environnement et de la santé humaine» ${ }^{79}$. Pour sa part, le Comité sénatorial permanent de l'Énergie, de l'Environnement et des Ressources naturelles a rendu public son rapport final en mars $2008^{80}$; or, il n'a suscité aucune autre

\footnotetext{
${ }^{76}$ Environnement Canada, Division des substances existantes, Aperçu du Programme des substances existantes, Canada, avril 2007, p. 8.

${ }^{77}$ Comité permanent de l'environnement et du développement durable, Examen quinquennal de la Loi canadienne sur la protection de l'environnement, 1999: combler les lacunes, avril 2007, Chambre des communes du Canada, Première session de la trente-neuvième législature, en ligne : < Chambre des communes - ENVI (39-1) - Examen quinquennal de la Loi canadienne sur la protection de l'environnement (1999): Combler les lacunes - Page couverture > (consulté le 26 mars 2010).

${ }^{78}$ Gouvernement du Canada, L'examen de la Loi canadienne sur la protection de l'environnement (1999); Réponse provisoire du gouvernement, octobre 2007, en ligne :

$<$ http://www.ec.gc.ca/RegistreLCPE/review/CR_Process/CR_rep-res/CR_rep-res.pdf> (consulté le 26 mars 2010).

${ }^{79}$ Id., p. 1 .

${ }^{80}$ Comité sénatorial permanent de l'énergie, de l'environnement et des ressources naturelles, Loi canadienne sur la protection de l'environnement (1999, ch. 33); Rx: Renforcez et appliquez avec soin, mars 2008, Deuxième session de la trente-neuvième législature, en ligne :
} 
réaction du gouvernement. Cet examen de la Loi permet de dresser un premier bilan de son application et notamment de la section $\mathrm{V}$ portant sur l'évaluation et la gestion des substances toxiques.

La présente partie a pour objet de formuler quelques pistes de réflexion quant aux modifications qui devraient être apportées à la Loi pour mieux encadrer le traitement réservé aux situations d'incertitude scientifique par les évaluateurs de risques et les décideurs publics. Ces situations s'avéreront nécessairement nombreuses particulièrement à l'endroit des substances chimiques catégorisées pour lesquelles les connaissances scientifiques demeureront souvent fragmentaires mais susceptibles d'évolution.

Deux remarques s'imposent d'entrée de jeu. D'une part, il est indéniable qu'au niveau mondial, le Canada a fait œuvre de pionnier en imposant un processus de catégorisation et de révision complète des substances commerciales et ce, plusieurs années avant la mise en œuvre du règlement REACH de l'Union européenne ${ }^{81}$. Nous croyons que la reconnaissance du principe de précaution et la nécessité d'instaurer une démarche prudente à l'endroit des risques incertains posés par les substances existantes ne font pas de doute à la lecture de la L.C.P.E. et des documents d'application étudiés précédemment ${ }^{82}$. D'ailleurs, le processus instauré par la L.C.P.E. pourrait permettre au Canada de s'illustrer sur la scène internationale par la prise de mesures concrètes pour contrer les effets de substances présentant un potentiel de toxicité, parfois avant que d'autres États ne soient prêts à le faire. La décision du gouvernement canadien de prendre des mesures visant le Bisphénol A, une substance dont la nocivité pour l'être humain est entourée d'incertitudes sur le plan scientifique, est révélatrice à cet égard ${ }^{83}$.

D'autre part, les critiques formulées à ce jour à l'endroit de la L.C.P.E. concernent bien davantage sa mise en œuvre et les ressources qui y sont associées que la teneur de ses

$<$ http://www.parl.gc.ca/39/2/parlbus/commbus/senate/com-f/enrg-f/rep-f/rep06mar08-f.htm $>$ (consulté le 26 mars 2010).

${ }^{81}$ Règlement (CE) 1907/2006 du Parlement européen et du Conseil du 18 décembre 2006 concernant l'enregistrement, l'évaluation et l'autorisation des substances chimiques.

${ }^{82}$ Voir supra, pp 18 à 27.

${ }^{83}$ Voir Gouvernement du Canada, «Le gouvernement du Canada protège les familles par la réglementation du bisphénol A», en ligne: <http://www.chemicalsubstanceschimiques.gc.ca/challenge-defi/batch-lot2/bisphenol-a/index-fra.php> (consulté le 9 avril 2010). 
dispositions et l'intention initiale du gouvernement fédéral au moment de son adoption ${ }^{84}$. Concrètement, il s'agit de savoir si l'éléphant a accouché d'une souris et si, outre quelques dossiers bien médiatisés à l'occasion desquels le Canada pourra se targuer d'être un chef de file mondial, les mesures mises en place s'avéreront suffisantes pour initier un virage significatif. Le but recherché par la Loi est d'instaurer un processus obligatoire de gestion des produits chimiques pouvant présenter un potentiel de toxicité pour la santé humaine ou l'environnement, dans un délai raisonnable. Ce processus devrait être assorti de mesures de contrôle et de sanctions efficaces. Il faut assurer la limitation et parfois l'élimination obligatoire des produits chimiques pouvant être dangereux, même en l'absence de certitude scientifique. La commande est claire. Il serait malheureux que l'exercice ne se traduise dans les faits que par une légitimation de la situation antérieure, et que l'absence de certitude scientifique absolue quant à la connaissance des risques ne conduise une fois de plus à l'inaction dans la plupart des cas.

Pour éviter ces écueils, il est essentiel de chercher à améliorer la portée de la Loi. En considérant plus spécifiquement le traitement réservé aux risques incertains, nos réflexions s'articuleront autour de trois objectifs primordiaux qui se sont dégagés des consultations tenues à l'occasion de l'examen de la Loi et des travaux du Parlement et qui devraient orienter, à notre avis, les réformes législatives post-révision. Il faudrait ainsi opérationnaliser le principe de précaution et diminuer la discrétion administrative (3.1), instaurer le principe de la responsabilité de l'industrie dans la démonstration de l'innocuité des substances (3.2) et assurer la vérification de la preuve scientifique et l'indépendance des évaluateurs gouvernementaux (3.3).

\footnotetext{
${ }^{84}$ H. Wilkins et E. MacDonald, «The Lion that Squeaked: CEPA, Mercury, and the Need for Better Regulation and Enforcement», (2009) 19 J.E.L.P. 167. Voir aussi : Comité permanent de l'environnement et du développement durable, Examen quinquennal de la Loi canadienne sur la protection de l'environnement, 1999: combler les lacunes, avril 2007, Chambre des communes du Canada, Première session de la trenteneuvième législature, en ligne : < Comités de la Chambre des communes - ENVI (39-1) - Examen quinquennal de la Loi canadienne sur la protection de l'environnement (1999) : Combler les lacunes - Page couverture $>$ aux pp. 4-5, (consulté le 26 mars 2010) et Comité sénatorial permanent de l'énergie, de l'environnement et des ressources naturelles, Loi canadienne sur la protection de l'environnement (1999, ch. 33); Rx: Renforcez et appliquez avec soin, mars 2008, Deuxième session de la trente-neuvième législature, en ligne : <http://www.parl.gc.ca/39/2/parlbus/commbus/senate/com-f/enrg-f/rep-f/rep06mar08$\underline{\text { f.htm> }}$ aux pp. 3 à 6, (consulté le 26 mars 2010).
} 


\section{Opérationnaliser le principe de précaution et diminuer la discrétion administrative}

Le principe de précaution fait l'objet d'une section spécifique du rapport du CPEDD. Ce dernier suggère entre autres une reformulation du principe de précaution qui permettrait possiblement d'en simplifier l'interprétation dans le contexte de la Loi. Il explique aussi que beaucoup de témoins ont proposé, lors des consultations sur l'examen de la L.C.P.E., d'augmenter le nombre de mesures obligatoires à prendre en vertu des dispositions de la Loi portant sur le principe de précaution, notamment en améliorant les échéances, et en tenant compte des populations et des écosystèmes vulnérables ${ }^{85}$. Le rapport du Comité sénatorial ne contient pas de chapitre spécifique portant sur le principe de précaution ou le traitement de l'incertitude scientifique; il aborde néanmoins de façon indirecte ces questions par une étude de cas, soit celle des composés perfluorés (notamment les ACPF et les APFO) employés dans de nombreux procédés industriels et produits de consommation et dont les caractéristiques de persistance, bioaccumulation et toxicité pour l'environnement sont récemment devenues une source d'inquiétude croissante pour la communauté scientifique internationale ${ }^{86}$.

Ces rapports attestent que l'application du «principe de prudence» s'avère obligatoire pour les ministres de l'Environnement et de la Santé lorsqu'ils procèdent à une évaluation préalable ou à l'évaluation d'une substance prioritaire. L'article 76.1 L.C.P.E. est clair à cet égard; il pourrait donc théoriquement servir de fondement à une action visant le contrôle judiciaire du processus décisionnel du gouvernement en cas de non respect. Mais le principe de précaution est-il rendu suffisamment opérationnel par la L.C.P.E pour jouer

\footnotetext{
${ }^{85}$ Comité permanent de l'environnement et du développement durable, Examen quinquennal de la Loi canadienne sur la protection de l'environnement, 1999: combler les lacunes, avril 2007, Chambre des communes du Canada, Première session de la trente-neuvième législature, en ligne : $<\underline{\text { Comités de la }}$ Chambre des communes - ENVI (39-1) - Examen quinquennal de la Loi canadienne sur la protection de l'environnement (1999): Combler les lacunes - Page couverture $>$ aux pp. 26 à 32, (consulté le 26 mars 2010).

86 Comité sénatorial permanent de l'énergie, de l'environnement et des ressources naturelles, Loi canadienne sur la protection de l'environnement (1999, ch. 33); Rx : Renforcez et appliquez avec soin, mars 2008, Deuxième session de la trente-neuvième législature, en ligne :

<http://www.parl.gc.ca/39/2/parlbus/commbus/senate/com-f/enrg-f/rep-f/rep06mar08-f.htm> aux pp. 29 à 43, (consulté le 26 mars 2010).
} 
ce rôle, de par les termes employés pour en préciser les contours et les quelques documents gouvernementaux explicatifs qui ont été préparés?

À l'instar du CPEDD, nous croyons que c'est par l'ajout d'obligations précises dans le texte de la loi que le principe de précaution saura trouver un contenu plus spécifique. Notamment, les seuils applicables pour déclencher la prise en compte du principe, soit l'existence d'un «risque de dommage grave ou irréversible» et l'absence de «certitude scientifique absolue» nécessitent une connaissance nuancée de la preuve scientifique disponible qu'il pourrait être très difficile d'apprécier judiciairement. Il est connu que les standards de la connaissance scientifique et les fardeaux de preuve juridiques ne font pas nécessairement bon ménage ${ }^{87}$. Mieux vaut donc, lorsque c'est possible, articuler en amont des échelles et critères au moyen de normes législatives ou réglementaires pour préciser le jugement scientifique quant à l'existence de ces seuils que de laisser une trop large discrétion qui empêchera, à l'aval, un réel contrôle judiciaire de la décision administrative $^{88}$. Ceci est d'autant plus vrai qu'il peut exister un risque élevé de multiplication des poursuites contre le gouvernement par l'industrie chimique, si la commercialisation de nombreuses substances est restreinte sans qu'un lien de causalité clair soit établi entre ces substances et des dommages identifiés.

Plusieurs propositions de modifications à apporter à la Loi vont dans ce sens. Par exemple, le CPEDD recommande de «modifier le préambule de la LCPE 1999 pour prendre acte de la nécessité de protéger les plus vulnérables dans notre société, notamment les enfants, et de modifier la Partie 5 pour inclure des dispositions semblables à celles de la Loi sur les produits antiparasitaires prévoyant de tenir compte des groupes vulnérables dans l'évaluation des risques, y compris un facteur de sécurité dix fois

\footnotetext{
${ }^{87}$ Voir en particulier : L.A. Reynolds, «The Era of Juriscience : Investigating the Relationship Between Science, Law and the Environment», (1995) C.J.A.L.P. 61, 85-88.

${ }^{88}$ «To date, [the precautionary principle] has been adverted to as a discretionary consideration or background interpretive canon. However, there is also growing evidence of a judicial appetite to engage with the principle in a more systematic doctrinal fashion [...]. Of course, whether and to what extent this aspiration can be realized depends on whether the precautionary principle can be rendered sufficiently coherent and predictable to serve as a basis for judicial decision-making» : C. Tollefson et J. Thornback, «Litigating the Precautionary Principle in Domestic Courts» (2008) 19 J.E.L.P. 33, 34.
} 
supérieur pour les enfants, le cas échéant» ${ }^{89}$. Le Comité précise que «l'évaluation des risques tient d'ores et déjà compte des populations vulnérables, mais on ne sait pas très bien comment cette information est, en fait, employée» ${ }^{90}$. Il conclut que cela devrait être explicite dans la Loi, et qu'un surcroît de précaution est justifié, notamment en raison des préoccupations grandissantes de la communauté scientifique concernant les effets néfastes que pourraient avoir les substances chimiques sur le développement intellectuel des enfants $^{91}$. Les auteurs du rapport recommandent en conséquence que le gouvernement ajoute les tests de neurotoxicité développementale à ceux exigés dans le Règlement sur les renseignements concernant les substances nouvelles et qu'il les exige aussi des fabricants dans le cas des substances existantes. ${ }^{92}$

Par ailleurs, concernant les écosystèmes vulnérables, le CPEDD estime que le préambule de la L.C.P.E. devrait contenir un paragraphe énonçant la nécessité de les protéger. Vu que les eaux des Grands Lacs s'avèrent grandement menacées en raison de la contamination par les substances toxiques, le Comité mentionne que le gouvernement devrait envisager toutes les options disponibles en vertu de la Loi pour prévoir un règlement d'application limitée destiné à protéger cette région sensible ${ }^{93}$. Nous souscrivons à cette recommandation.

De plus, soulignons que le principe de précaution inclus généralement des considérations relatives au temps d'inaction par rapport au temps d'accumulation des éléments de preuve. Ainsi, l'ajout de délais dans la Loi là où il n'y en a pas permettrait de contrer l'inaction gouvernementale, notamment pour assurer la gestion rapide des substances à

\footnotetext{
${ }^{89}$ Comité permanent de l'environnement et du développement durable, Examen quinquennal de la Loi canadienne sur la protection de l'environnement, 1999: combler les lacunes, avril 2007, Chambre des communes du Canada, Première session de la trente-neuvième législature, en ligne : $<$ Comités de la Chambre des communes - ENVI (39-1) - Examen quinquennal de la Loi canadienne sur la protection de l'environnement (1999): Combler les lacunes - Page couverture > à la p. 31, (consulté le 26 mars 2010). Voir aussi: T. McClenaghan, K. Cooper, L. Vanderlinden, P. Muldoon, A. Abelsohn, K. Khatter et K. Keenan, «Environmental Standard Setting and Children's Health in Canada : Injecting Precaution into Risk Assessment», (2003) 12 J.E.L.P. 245.

${ }^{90}$ Id., p. 29.

${ }^{91} I d$.

${ }^{92} I d$. p. 24

${ }^{93}$ Id., pp. 31-32.
} 
l'endroit desquelles les risques demeurent incertains. L'expérience a démontré que des délais précis dans la Loi peuvent être respectés: l'exercice de catégorisation des substances de la L.I.S. a été complété en sept ans comme prévu, l'évaluation préalable des 200 substances les plus prioritaires après catégorisation est en bonne voie d'être réalisée dans le délai de trois ans que le gouvernement s'est lui-même imposé ${ }^{4}$. Le délai d'évaluation et de traitement de toutes les autres substances ayant été catégorisées et de celles qui seront ajoutées à la Liste des substances prioritaires n'est cependant pas fixé par la Loi. Des délais devraient être spécifiés à cet égard. Également, il demeure qu'un risque réel ou potentiel n'est pas contré tant qu'il n'est pas géré, et que la Loi devrait en conséquence être modifiée pour éviter la discrétion administrative dans le choix du moment pour donner suite à l'évaluation des risques. Le CPEDD suggère les modifications suivantes :

- Dans le cas d'une évaluation préalable établissant qu'une substance est toxique, fixer à deux ans le délai compris entre le début de l'évaluation préalable et celui d'un plan de mise en œuvre.

- Dans le cas d'une évaluation préalable établissant la nécessité d'effectuer une évaluation complète de la Liste des substances d'intérêt prioritaire, fixer à cinq ans le délai compris entre le début de l'évaluation préalable et celui du plan de mise en cuvre. ${ }^{95}$

L'imposition de délais impératifs par voie législative dans l'évaluation et la gestion des substances catégorisées risque d'entraîner en contrepartie une plus grande incertitude dans les résultats des évaluations ${ }^{96}$. Cependant, cette incertitude ne devrait pas se traduire par

94 Gouvernement du Canada, «Plan de gestion des produits chimiques», en ligne : $<$ http://www.chemicalsubstanceschimiques.gc.ca/plan/index-fra.php $>$ (consulté le 2 avril 2010).

95 Comité permanent de l'environnement et du développement durable, Examen quinquennal de la Loi canadienne sur la protection de l'environnement, 1999: combler les lacunes, avril 2007, Chambre des communes du Canada, Première session de la trente-neuvième législature, en ligne : $<\underline{\text { Comités de la }}$ Chambre des communes - ENVI (39-1) - Examen quinquennal de la Loi canadienne sur la protection de l'environnement (1999): Combler les lacunes - Page couverture > à la p. 29, (consulté le 26 mars 2010).

96 «Ce que j'essaie de dire, essentiellement, c'est que si l'on me donne un délai de six semaines, de six mois ou de six ans, nous allons faire ce que nous avons à faire dans ce délai. C'est notre travail. Ce qui augmente à mesure que le temps alloué diminue, c'est le degré de variabilité ou d'incertitude qui entoure une évaluation. Si nous avons plus de temps, nous avons la capacité de faire plus de recherches scientifiques et de parvenir à une plus grande certitude. Avec moins de temps, si l'information n'est pas disponible, il y a plus d'incertitude dans nos évaluations» : témoignage de Paul Grover, directeur général, Programme de la 
une absence de prise de mesures à l'endroit des substances douteuses, si est aussi parallèlement appliqué le principe de la responsabilité de l'industrie dans la démonstration de l'innocuité des substances, comme nous le verrons dans la prochaine section.

\section{Instaurer le principe de la responsabilité de l'industrie dans la démonstration de}

\section{l'innocuité des substances}

De longue date, des débats ont entouré la question de la responsabilité du producteur dans le domaine des substances chimiques, laquelle est fortement liée au principe de précaution. Qui, du gouvernement ou de l'industrie, doit fournir les données et pour ce faire, réaliser les études de risques associées aux substances? Qui doit en assumer les coûts? Comment en vérifier la qualité et l'exactitude lorsqu'elles proviennent des industries? Ces questions nous apparaissent, en effet, primordiales. Suite à la catégorisation, le gouvernement a lancé un « défi » encourageant les industries à fournir des informations additionnelles sur les 200 substances hautement prioritaires identifiées durant l'exercice, à défaut de quoi il s'est dit prédisposé à conclure à leur caractère toxique ${ }^{97}$. Cette application du principe de responsabilité du producteur s'inscrit dans le sens d'une prudence accrue.

Nous sommes d'avis cependant que le défi post catégorisation ne doit pas constituer une application isolée de cette manière de procéder. Un cadre d'orientation plus global devrait être élaboré afin de clarifier les paramètres permettant de départager les responsabilités de chacun dans le domaine des substances toxiques. À l'instar du CPEDD, nous croyons que « le principe selon lequel l'industrie devrait être tenue de persuader le gouvernement de l'innocuité d'une substance, actuelle ou nouvelle, (en fait, du risque acceptable qu'elle

sécurité des milieux, ministère de la Santé, 17 octobre 2006, rapporté à: Comité permanent de l'environnement et du développement durable, Examen quinquennal de la Loi canadienne sur la protection de l'environnement, 1999 : combler les lacunes, avril 2007, Chambre des communes du Canada, Première session de la trente-neuvième législature, en ligne : $<$ Comités de la Chambre des communes - ENVI (39-1) Examen quinquennal de la Loi canadienne sur la protection de l'environnement (1999): Combler les lacunes - Page couverture > à la p. 27, (consulté le 26 mars 2010).

${ }^{97} \mathrm{Ce}$ « défi » vise des lots de 15 à 30 substances à tous les 3 mois, pour une période totale de 2-3 ans. «Gouvernement du Canada, Plan de gestion des produits chimiques, Défi à l'industrie», en ligne :

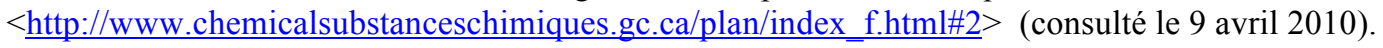


présente), est bon et devrait être inscrit dans la Loi. ${ }^{98}$ Pourraient servir de modèle les dispositions sur le fardeau de persuasion, que l'on retrouve dans la Loi sur les produits antiparasitaires $^{99}$, dans la L.C.P.E. et le Règlement sur les renseignements concernant les substances nouvelles (substances chimiques et polymères), ${ }^{100}$ concernant les substances nouvelles ou dans le texte du programme européen $\mathrm{REACH}^{101}$. Cette façon de procéder devrait être étendue à toutes les substances assujetties à l'évaluation préalable, puisqu'elles ont fait l'objet d'un premier examen par les évaluateurs du gouvernement et qu'elles remplissent les critères retenus pour la catégorisation. Il est donc légitime de présumer qu'elles sont dommageables pour l'environnement ou la santé humaine, parce qu'elles sont persistantes ou bioaccumulables et présentent une toxicité intrinsèque ou parce que les êtres humains y sont fortement exposés. L'absence de production d'une preuve contraire et crédible devrait entraîner systématiquement une conclusion de risques inacceptables, ce qui serait de nature à raccourcir les délais d'évaluation, la prise de mesures de gestion des risques et à assurer une réelle application du principe de précaution. Rien n'empêcherait par ailleurs le gouvernement de réviser sa décision si de nouvelles données scientifiques s'avéraient disponibles.

Il est certain que l'enjeu entourant la production de la preuve demeure important et que toute tentative visant à opérer un «renversement du fardeau de la preuve» doit être

\footnotetext{
${ }^{98}$ Comité permanent de l'environnement et du développement durable, Examen quinquennal de la Loi canadienne sur la protection de l'environnement, 1999: combler les lacunes, avril 2007, Chambre des communes du Canada, Première session de la trente-neuvième législature, en ligne :

$<$ http://cmte.parl.gc.ca/Content/HOC/committee/391/envi/reports/rp2614246/envirp05/05-rep-f.htm >, p. 12.

${ }^{99}$ L.C. 2002, c. 28 , alinéa 7(6)a) : «Lors des évaluations a) il incombe au demandeur de convaincre le ministre que la valeur du produit et les risques sanitaires et environnementaux qu'il présente sont acceptables $[\ldots] »$.

100 Règlement sur les renseignements concernant les substances nouvelles (substances chimiques et polymères), DORS/ 2005-247.

${ }_{101}$ Règlement (CE) 1907/2006 du Parlement européen et du Conseil du 18 décembre 2006 concernant l'enregistrement, l'évaluation et l'autorisation des substances chimiques. Ce règlement entrait en vigueur en juin 2007, sauf certaines dispositions en 2008 et 2009. Le règlement exige des fabricants et importateurs qu'ils soumettent des demandes d'enregistrement pour leurs produits et qu'ils fournissent les résultats de tests, des évaluations de risques et des stratégies de gestion des risques par rapport aux substances pour lesquelles ils soumettent leurs demandes.
} 
entourée de garanties ${ }^{102}$. En vertu de la L.C.P.E., la démarche d'évaluation et d'appréciation des risques demeure entre les mains des scientifiques du gouvernement qui sont responsables de décider si une substance s'avère toxique ou potentiellement toxique pour l'environnement ou la santé humaine. La recherche d'informations et de données disponibles est clairement imputée au gouvernement. Les dispositions de la Loi prévoient cependant que le gouvernement peut à cet égard exiger des renseignements et des études de la part de l'industrie ${ }^{103}$, qui sont à l'heure actuelle limités aux quantités et aux usages faits des substances mais qui pourraient aussi «porter si nécessaire sur des essais de toxicité additionnels» ${ }^{104}$. La Loi pourrait à notre avis être amendée pour prévoir explicitement qu'à défaut de présentation de preuves scientifiques crédibles, lors de l'évaluation préalable d'une substance catégorisée, démontrant de façon raisonnable qu'elle ne comporte pas de risque inacceptable pour l'environnement ou la santé humaine, les ministres sont fondés à conclure à son caractère toxique.

\section{Assurer la vérification de la preuve scientifique et l'indépendance des évaluateurs}

Les étapes du processus d'évaluation scientifique de risques présentent un grand intérêt en matière d'application du principe de précaution. En soi, «[1]'évaluation des risques est objective, fondée sur la science et n'est pas dépendante de considérations

\footnotetext{
102 «En première analyse, cette recommandation [le renversement du fardeau de la preuve] pourrait donner lieu à des interprétations assez perverses. Premièrement, il est impossible de garantir la sécurité absolue - en fait, faire la preuve du contraire - et, deuxièmement, le fait de confier au secteur privé le soin de faire la preuve de la sécurité reviendrait un peu à charger le loup de la bergerie. Ce n'est pas, bien entendu, ce dont il était question. Il y a plusieurs façons d'interpréter la notion de «fardeau de la preuve» qui répartissent le fardeau [...] du processus d'évaluation différemment entre les autorités et l'industrie» : Comité permanent de l'environnement et du développement durable, Examen quinquennal de la Loi canadienne sur la protection de l'environnement, 1999 : combler les lacunes, avril 2007, Chambre des communes du Canada, Première session de la trente-neuvième législature, en ligne :

$<$ http://cmte.parl.gc.ca/Content/HOC/committee/391/envi/reports/rp2614246/envirp05/05-rep-f.htm >, p. 10 (consulté le 26 mars 2010).

${ }^{103}$ Articles 46 et 71 L.C.P.E.

${ }^{104}$ Comité permanent de l'environnement et du développement durable, Examen quinquennal de la Loi canadienne sur la protection de l'environnement, 1999: combler les lacunes, avril 2007, Chambre des communes du Canada, Première session de la trente-neuvième législature, en ligne :

$<$ http://cmte.parl.gc.ca/Content/HOC/committee/391/envi/reports/rp2614246/envirp05/05-rep-f.htm >, p. 12 (consulté le 26 mars 2010).
} 
socioéconomiques $»^{105}$. Cependant, on ne retrouve pas dans la L.C.P.E. de dispositions relatives à la composition des groupes d'évaluateurs, à la durée de leur mandat, à leur expertise et à leur indépendance, tel que c'est le cas par exemple au Canada dans la Loi sur les espèces en péril ${ }^{106}$. Il nous semble pourtant que de telles garanties sont essentielles afin d'assurer objectivement un processus scientifique de qualité et indépendant. De plus, le paragraphe 90(1) de la L.C.P.E. laisse place à la discrétion politique au moment d'inscrire une substance sur la liste des substances toxiques. Certes, c'est «sur recommandation des ministres » que le gouverneur en conseil prendra sa décision, mais il devra "être convaincu qu'une substance est toxique» pour l'inscrire à l'annexe I. Le terme « convaincu » ainsi que l'absence de l'expression «potentiellement toxique » qu'on retrouve dans les autres articles sur l'évaluation des substances ouvrent la porte à une discrétion indue des décideurs dans un processus dont seules des considérations de nature scientifique doivent en principe fonder les conclusions. La Loi devrait, à notre avis, être modifiée pour bien traduire l'idée que toutes les substances considérées toxiques aux termes d'une évaluation scientifique des risques doivent de fait être inscrites à l'Annexe 1, sans qu'aucun arbitraire politique ne puisse potentiellement intervenir à un quelconque moment dans ce processus administratif.

Par ailleurs, le CPEDD propose au gouvernement de demander à des tiers de vérifier les données fournies par les personnes identifiées en vertu des articles 46 ou 71 de la Loi. Le comité parlementaire fait valoir qu'il est essentiel que le résultat de l'évaluation scientifique puisse résister à un examen et qu' «il est primordial de recourir aux ouvrages examinés par des pairs pour obtenir de l'information et d'exiger que les entreprises qui fournissent des données emploient les meilleures méthodes de laboratoire reconnues» ${ }^{107}$.

\footnotetext{
105 Environnement Canada, Division des substances existantes, Aperçu du Programme des substances existantes, Canada, avril 2007, p. 9.

${ }^{106}$ L.C. 2002, c. 29. Voir H. Trudeau et T. Leroux, «Conjurer un sort incertain : le principe de précaution et l'évaluation des espèces en péril au Canada», (2008) 87 R. du. B. Can. 161.

${ }^{107}$ Comité permanent de l'environnement et du développement durable, Examen quinquennal de la Loi canadienne sur la protection de l'environnement, 1999: combler les lacunes, avril 2007, Chambre des communes du Canada, Première session de la trente-neuvième législature, en ligne :

$<$ http://cmte.parl.gc.ca/Content/HOC/committee/391/envi/reports/rp2614246/envirp05/05-rep-f.htm $>$, p. 16 (consulté le 26 mars 2010).
} 
Le gouvernement semble accueillir favorablement une telle proposition ${ }^{108}$. D'ailleurs, les ministères de la Santé et de l'Environnement ont déclaré que «les évaluations font l'objet d'un examen par les pairs»; toutefois, comme le rapporte le Comité, «on ne sait pas très bien comment cela se passe» ${ }^{109}$.

Des entretiens conduits à l'intérieur de la Division des substances existantes d'Environnement Canada à l'hiver 2005 nous ont permis de recueillir les informations suivantes quant à la façon dont cheminent les dossiers d'évaluation des risques au sein de ce ministère. D'abord, une personne de la Division est désignée responsable de tous les volets de l'évaluation d'une substance. Son rôle est d'acquérir une connaissance approfondie de la substance, des sources de rejets et des effets sur l'environnement, en recherchant auprès des experts de la Division les compléments d'informations qui lui manquent pour en apprécier la toxicité éventuelle ${ }^{110}$. En cas de besoin, elle fait aussi appel à des expertises supplémentaires pour l'aider dans l'exécution de sa tâche ${ }^{111}$. Il peut s'agir de ressources gouvernementales externes à la Division, d'expertises provenant du milieu universitaire ou des informations issues des milieux industriels ${ }^{112}$. L'évaluateur se

${ }^{108}$ Gouvernement du Canada, L'examen de la Loi canadienne sur la protection de l'environnement (1999); Réponse provisoire du gouvernement, octobre 2007, en ligne : <http://www.ec.gc.ca/RegistreLCPE/review/CR_Process/CR_rep-res/CR_rep-res.pdf>, p. 13 (consulté le 26 mars 2010).

${ }^{109}$ Comité permanent de l'environnement et du développement durable, Examen quinquennal de la Loi canadienne sur la protection de l'environnement, 1999: combler les lacunes, avril 2007, Chambre des communes du Canada, Première session de la trente-neuvième législature, en ligne :

$<$ http://cmte.parl.gc.ca/Content/HOC/committee/391/envi/reports/rp2614246/envirp05/05-rep-f.htm >, p. 17 (consulté le 26 mars 2010).

110 Entretiens, 2005. «Divers types d'information servent à réaliser l'évaluation écologique d'une substance. Figurent parmi ceux-ci l'identité chimique, les propriétés physicochimiques, les utilisations industrielles, les rejets et la dégradation de la substance dans l'environnement, l'exposition possible à la substance dans ce milieu et l'écotoxicité potentielle de la substance» : Environnement Canada, Division des substances existantes et Division des substances nouvelles, Aperçu de l'évaluation écologique des substances en vertu de la Loi canadienne sur la protection de l'environnement (1999), Canada, juin 2007, p. 8.

${ }^{111}$ Cette manière de procéder correspond au Principe II du Cadre applicable aux avis en matière de sciences et de technologie préparé par le Gouvernement fédéral : Gouvernement du Canada, Cadre applicable aux avis en matière de sciences et de technologie : principes et lignes directrices pour une utilisation efficace des avis relatifs aux sciences et à la technologie dans le processus décisionnel du gouvernement, Industrie Canada, 2000, pp. 5-7.

112 Entretiens, 2005; Environnement Canada, « Évaluation des substances existantes : activités connexes »,

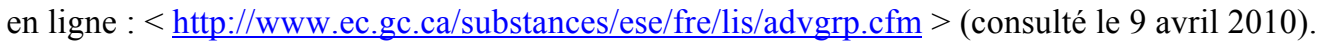


rapporte à un chef de section ou à un autre responsable de la supervision de l'évaluation, qui s'assure que les approches utilisées sont consistantes et qui vérifie le bien-fondé des conclusions du rapport sur la substance.

Plusieurs autres étapes jalonnent le processus d'évaluation. Celle de la présentation des résultats préliminaires à des groupes d'évaluateurs et de gestionnaires de risques de la Division, par exemple, permet de réviser l'ébauche de l'évaluation à l'interne. Un groupe de révision externe est aussi souvent formé à ce stade, groupe qui comprendra des experts émanant de différents secteurs : industries, universités, groupes environnementaux. Pourront aussi agir comme réviseurs externes les fonctionnaires impliqués dans la réglementation des substances toxiques aux États-Unis, en Europe ou ailleurs sur la scène internationale. Le dossier est ensuite approuvé par le directeur de la Division des substances existantes, qui le soumet au Comité de gestion de la L.C.P.E. ${ }^{113}$. Il est important de mentionner que les commentaires du public sont sollicités à plusieurs étapes du cheminement d'un dossier d'évaluation et de gestion d'une substance donnée ${ }^{114}$.

Ces étapes de révision à l'interne et à l'externe des données disponibles ne sont pas explicitement prévues dans la Loi. Le comité sénatorial dans son rapport sur l'examen de la L.C.P.E. soutient que le principal problème relatif à la Loi réside dans un manque de ressources et de volonté à l'appliquer. Le manque de ressources a aussi été identifié par le CPEDD et par plusieurs autres observateurs par le passé ${ }^{115}$. Ce manque est accentué par la

\footnotetext{
113 «Le Comité de gestion de la LCPE a vu le jour en vertu d'un protocole d'entente entre Environnement Canada et Santé Canada. Il est responsable des dispositions de la LCPE (1999) qui visent l'évaluation et la gestion des risques ainsi que la lutte contre les substances toxiques. Cette portée s'étend aux programmes des ministères qui traitent des substances nouvelles et présentes, des produits de la biotechnologie, de la collecte d'information, de la catégorisation et de l'examen préalable des substances de la liste intérieure des substances et de la liste des substances d'intérêt prioritaire. Le Comité voit également à l'élaboration, pour les substances toxiques d'options réglementaires et non réglementaires, de gestion et de contrôle. Il est formé de représentants d'Environnement Canada et de Santé Canada. », Environnement Canada / Santé Canada, Document d'orientation concernant la Loi canadienne sur la protection de l'environnement (1999) et le principe de prudence - Amélioration de la prise de décisions dans l'incertitude, p. 22.

${ }^{114}$ Cela correspond d'ailleurs au Principe V du Cadre applicable aux avis en matière de sciences et de technologie. Gouvernement du Canada, Cadre applicable aux avis en matière de sciences et de technologie : principes et lignes directrices pour une utilisation efficace des avis relatifs aux sciences et à la technologie dans le processus décisionnel du gouvernement, Industrie Canada, 2000, pp. 10-12.

${ }^{115}$ Voir entre autres Table ronde nationale sur l'environnement et l'économie, La gestion des substances potentiellement toxiques au Canada, 2001, Canada, Ottawa; Environnement Canada, Diagnostic, préparation pour l'examen parlementaire de la Loi canadienne sur la protection de l'environnement, 1999, 2004, Canada.
} 
rareté des experts scientifiques qualifiés ${ }^{116}$. Dans ce contexte, on peut légitimement se demander si les évaluateurs de risques au sein des deux ministères concernés auront un budget suffisant leur permettant d'assurer la vérification par des pairs des résultats de leurs analyses, surtout dans le contexte de l'évaluation post-catégorisation de centaines de substances potentiellement dangereuses.

En réponse à la recommandation du CPEDD d'attribuer davantage de ressources au Programme des substances existantes, le gouvernement répond qu'il a investi plus d'un demi-milliard de dollars au cours de l'année 2006-2007 dans la gestion de la qualité de l'air et des substances chimiques et qu'il « continuera à revoir les besoins en ressources pour mettre en œuvre efficacement la LCPE (1999) et les autres priorités de protection de l'environnement et de la santé»» ${ }^{117}$. Cela constitue à notre avis une réponse plutôt nébuleuse.

\section{CONCLUSION}

Bien que le principe de précaution constitue un principe fondamental de la L.C.P.E., il demeure que le gouvernement dispose d'une très large discrétion quant à l'interprétation qu'il souhaite lui prêter et l'application qu'il désire en faire. En effet, le degré de certitude déclenchant l'application du principe et la décision d'adopter des mesures de gestion des risques sont des paramètres qui nécessitent l'application de choix éthiques réalisés au cas par cas. Ils sont aussi fonction du rôle que souhaite et que peut adopter le gouvernement fédéral en matière de contrôle des substances toxiques. Au-delà de ces considérations, c'est aussi beaucoup du savoir scientifique disponible dont il s'agit et du constat que

\footnotetext{
${ }^{116}$ Environnement Canada. Diagnostic, préparation pour l'examen parlementaire de la Loi canadienne sur la protection de l'environnement, 1999, 2004, Canada, p. 30.

${ }^{117}$ Gouvernement du Canada, L'examen de la Loi canadienne sur la protection de l'environnement (1999); Réponse provisoire du gouvernement, octobre 2007, en ligne :

<http://www.ec.gc.ca/RegistreLCPE/review/CR_Process/CR_rep-res/CR_rep-res.pdf $>$, p. 21 (consulté le 26 mars 2010).
} 
celui-ci n'est pas complet et qu'il convient d'en combler les lacunes avec la prudence qui s'impose ${ }^{118}$.

Nos entretiens effectués auprès de fonctionnaires d'Environnement Canada en 2005 ont révélé que jusqu'à cette date, l'inscription du principe de précaution à la L.C.P.E. avait eu deux effets principaux sur les évaluations effectuées par la Division des substances existantes. D'une part, on mentionnait que l'approche recommandée était beaucoup plus globale qu'autrefois et reposait sur une évaluation de l'impact des substances dans l'environnement à court, à moyen et à long terme. D'autre part, l'accent mis sur la précaution permettait dorénavant aux évaluateurs et aux gestionnaires de risques de recommander puis de prendre des mesures préventives à l'endroit d'une substance qui semblait présenter un potentiel de toxicité pour l'environnement ou la santé humaine, alors même que l'évaluation n'était pas terminée, et donc d'agir efficacement beaucoup plus rapidement.

Il serait certainement pertinent de réaliser des études de cas quant à l'application du principe de précaution maintenant que certaines des évaluations préalables suivant le processus de catégorisation sont réalisées et qu'elles conduiront à la prise de mesures de gestion de risque. Cela permettrait d'évaluer avec davantage de précision si l'énonciation du principe de précaution dans la Loi canadienne sur la protection de l'environnement se traduit par un réel changement procédural et décisionnel ou s'il s'agit d'un simple exercice de rhétorique ${ }^{119}$.

Puisque la catégorisation des substances est désormais terminée, que le projet de loi sur le renouvellement de la L.C.P.E. est sous étude et que le programme européen REACH est entré en vigueur, il sera certes important de suivre les développements qui auront lieu en matière d'évaluation et de gestion des substances chimiques dans les années à venir, au Canada comme ailleurs dans le monde.

\footnotetext{
${ }^{118}$ Comité permanent de l'environnement et du développement durable, Examen quinquennal de la Loi canadienne sur la protection de l'environnement, 1999: combler les lacunes, avril 2007, Chambre des communes du Canada, Première session de la trente-neuvième législature, en ligne : <Comités de la Chambre des communes - ENVI (39-1) - Examen quinquennal de la Loi canadienne sur la protection de l'environnement (1999): Combler les lacunes - Page couverture >, p. 2 (consulté le 26 mars 2010).
} 


\section{BIBLIOGRAPHIE}

\section{Doctrine juridique}

J. Cameron, «Future Directions in International Environmental Law: Precaution, Integration and Non-state Actors » (1996) 19 Dalhousie Law Journal 122.

J. Cameron et J. Abouchar, «The Precautionary Principle : A Fundamental Principle of Law and Policy for the Protection of the Global Environment » (1991) 14 Boston College International \& Comparative Law Review 1.

R. Daigneault, «La L.C.P.E. 1999 : portée et enjeux», Formation permanente, Barreau du Québec, Développements récents en droit de l'environnement (2000), Cowansville, Éditions Yvon Blais, 2000.

N. de Sadeleer, Les principes du pollueur-payeur, de prévention et de précaution, Bruxelles, Bruylant, 1999.

D. Freestone et E. Hey, eds., The Precautionary Principle and International Law. The Challenge of Implementation, The Hague, London, Boston, Kluwer International Law, 1996.

E. Hey, «The Precautionary Concept in Environmental Policy and Law : Institutionalizing Caution » (1992) 4 The Georgetown Int'l Envtl. Law Review 303.

J. E. Hickey et V. R. Walker, « Refining the Precautionary Principle in Environmental Law » (1995) 14 Virginia Environmental Law Journal 423.

P. Kourilsky et G. Viney, Le principe de précaution, Paris, Éditions Odile Jacob, 2000.

T. McClenaghan, K. Cooper, L. Vanderlinden, P. Muldoon, A. Abelsohn, K. Khatter et K. Keenan, «Environmental Standard Setting and Children's Health in Canada : Injecting Precaution into Risk Assessment», (2003) 12 J.E.L.P. 245.

\footnotetext{
119 VanderZwaag, David, « The Precautionary Principle in Environmental Law and Policy: An Elusive Rhetoric and First Embraces », 8 J.E.L.P. 355
} 
O.McIntyre et T. Mosedale, "The Precautionary Principle as a Norm of Customary International Law» (1997) 9 Journal of Environmental Law 221.

L.A. Reynolds, «The Era of Juriscience : Investigating the Relationship Between Science, Law and the Environment», (1995) C.J.A.L.P. 61.

C. Tollefson et J. Thornback, «Litigating the Precautionary Principle in Domestic Courts» (2008) 19 J.E.L.P. 33.

H. Trudeau, «Du droit international au droit interne ; l'émergence du principe de précaution en droit de l'environnement», (2003) 28 Queen's Law Journal 455.

H. Trudeau et T. Leroux, «Conjurer un sort incertain : le principe de précaution et l'évaluation des espèces en péril au Canada», (2008) 87 R. du. B. Can. 161.

D. VanderZwaag, La LCPE et le principe ou l'approche précaution, Examen de la LCPE: Document d'élaboration des enjeux 18, Canada, Ministre des Approvisionnement et Services, 1994.

D. VanderZwaag, «The Precautionary Principle in Environmental Law and Policy: An Elusive Rhetoric and First Embraces », 8 J.E.L.P. 355.

H. Wilkins et E. MacDonald, «The Lion that Squeaked: CEPA, Mercury, and the Need for Better Regulation and Enforcement», (2009) 19 J.E.L.P. 167.

T. Williams, «Élimination virtuelle de la pollution résultant du rejet des substances toxiques», 26 juillet 2006, Bibliothèque du Parlement, Canada, en ligne :

$<$ http://www2.parl.gc.ca/content/lop/researchpublications/prb0626-f.htm> .

\section{Documents et rapports gouvernementaux et d'organisations internationales}

Gouvernement du Canada, «Plan de gestion des produits chimiques», en ligne: $<\underline{\text { http://www.chemicalsubstanceschimiques.gc.ca/plan/index-fra.php }>}$

Environnement Canada, «La Loi canadienne sur la protection de l'environnement, 1999 (LCPE 1999) et l'évaluation des substances actuelles », en ligne :

$<\underline{\text { http://www.ec.gc.ca/RegistreLCPE/gene_info/fact 08.cfm }>}$ 
Environnement Canada, «Inventaire national des rejets de polluants», en ligne: $<\underline{\text { http://www.ec.gc.ca/inrp-npri/default.asp?lang=Fr\&n=4A577BB9-1 }>}$

Gouvernement du Canada, L'examen de la Loi canadienne sur la protection de l'environnement (1999); Réponse provisoire du gouvernement, octobre 2007, en ligne : $<\underline{\text { http://www.ec.gc.ca/RegistreLCPE/review/CR_Process/CR_rep-res/CR_rep-res.pdf }>}$

Gouvernement du Canada, Mesures législatives sur la protection de l'environnement conçues pour l'avenir - Une LCPE renouvelée, Ottawa: Ministre des Approvisionnements et Services, 1995

Environnement Canada, « Liste des substances d'intérêt prioritaire », en ligne :

$<\underline{\text { http://www.ec.gc.ca/RegistreLCPE/subs_list/Priority.cfm }>}$

Environnement Canada, «Aperçu du Programme des substances existantes à Environnement Canada $\gg, \quad$ en ligne : < http://www.ec.gc.ca/substances/ese/fre/overview.cfm $>$

Organisation de coopération et de développement économiques, «Sécurité des produits chimiques », en

ligne :

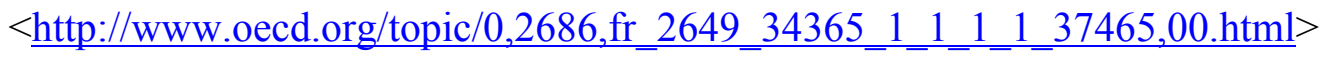

O.E.C.D. «eChemPortal : The Global Portal to Information on Chemical Substances», en ligne : http://webnet3.oecd.org/echemportal/

Environnement Canada, « Guide technique », en ligne :

$<\underline{\text { http://www.ec.gc.ca/substances/ese/fre/pesip/psl2manual.cfm }}>$

Gouvernement du Canada, " Projet pilote pour l'évaluation préalable », en ligne : $<$ http://www.chemicalsubstanceschimiques.gc.ca/about-apropos/assess-eval/projet-pilotproject/index-fra.php>

Comité sénatorial permanent de l'énergie, de l'environnement et des ressources naturelles, Loi canadienne sur la protection de l'environnement (1999, ch. 33); Rx: Renforcez et appliquez avec soin, mars 2008, Deuxième session de la trente-neuvième législature, en ligne : <http:/www.parl.gc.ca/39/2/parlbus/commbus/senate/com-f/enrg$\underline{\text { f/rep-f/rep06mar08-f.htm }>}$ 
Environnement Canada / Santé Canada, Document d'orientation concernant la Loi canadienne sur la protection de l'environnement (1999) et le principe de prudence: amélioration de la prise de décisions dans l'incertitude

Commission mixte internationale, Septième rapport biennal en vertu de l'Accord de 1978 relatif à la qualité de l'eau dans les Grands Lacs présenté aux Gouvernements des ÉtatsUnis et du Canada et aux Gouvernements des États et de la Province du Bassin des Grands Lacs, 1994, en ligne : < http://www.ijc.org/php/publications/html/7brf.html>

Groupe de travail sur l'élimination virtuelle, A Strategy for Virtual Elimination of Persistent Toxic Substances (Volumes 1 et 2), Rapport final présenté à la Commission mixte internationale, Windsor, Ontario, août 1993

Chambre des communes du Canada, Comité permanent de l'environnement et du développement durable, Notre santé en dépend! Vers la prévention de la pollution: Examen de la LCPE, Ottawa, Service des publications, 1995

Gouvernement du Canada, Mesures législatives sur la protection de l'environnement conçues pour l'avenir - Une LCPE renouvelée, Examen de la LCPE: Réponse du gouvernement, Ottawa, Ministre des Approvisionnements et Services, 1995

Gouvernement du Canada, Bureau du Conseil privé, Cadre d'application de la précaution dans un processus décisionnel scientifique en gestion du risque, 2003, en ligne: $<\underline{\text { http://www.pco- }}$

bcp.gc.ca/index.asp?lang=fra\&page $=$ information $\&$ sub $=$ publications $\&$ doc $=$ precaution/pre caution_f.htm >

Environnement Canada, Aperçu de l'évaluation écologique des substances en vertu de la Loi canadienne sur la protection de l'environnement (1999), Canada, juin 2007

Comité permanent de l'environnement et du développement durable, Examen quinquennal de la Loi canadienne sur la protection de l'environnement, 1999 : combler les lacunes, avril 2007, Chambre des communes du Canada, Première session de la trenteneuvième législature, en ligne : $<$ Comités de la Chambre des communes - ENVI (39-1) Examen quinquennal de la Loi canadienne sur la protection de l'environnement (1999): Combler les lacunes - Page couverture $>$ 
Gouvernement du Canada, «Le gouvernement du Canada protège les familles par la réglementation $\mathrm{du}$ bisphénol A», en ligne : $<$ http://www.chemicalsubstanceschimiques.gc.ca/challenge-defi/batch-lot-2/bisphenola/index-fra.php $>$

Gouvernement du Canada, Cadre applicable aux avis en matière de sciences et de technologie : principes et lignes directrices pour une utilisation efficace des avis relatifs aux sciences et à la technologie dans le processus décisionnel du gouvernement, Industrie Canada, 2000

Table ronde nationale sur l'environnement et l'économie, La gestion des substances potentiellement toxiques au Canada, 2001, Canada, Ottawa

Environnement Canada, Diagnostic, préparation pour l'examen parlementaire de la Loi canadienne sur la protection de l'environnement, 1999, 2004, Canada

\section{Documents et rapports d'organisations non gouvernementales}

Défense environnementale, «Une nation toxique : Rapport sur la pollution chez les Canadiens », 2005,

$<\underline{\text { http://www.toxicnation.ca/files/toxicnation/report/PATN_FrenchWeb.pdf }}>$

Défense environnementale, « Des enfants empoisonnés - une nation toxique : Rapport sur la pollution chez les familles canadiennes», 2006, $<$ http://www.toxicnation.ca/files/toxicnation/report/PCTN_French.pdf $>$

Défense environnementale, «Une nation toxique sur la Colline du Parlement: Rapport sur la pollution chez quatre politiciens canadiens», 2007, en ligne : $<$ http://www.environmentaldefence.ca/toxicnation/report/TN-

PARLIAMENTHILL fr.pdf> 
